NBER WORKING PAPER SERIES

\title{
FUTURE PATHS OF ELECTRIC VEHICLE ADOPTION IN THE UNITED STATES: PREDICTABLE DETERMINANTS, OBSTACLES AND OPPORTUNITIES
}

\author{
James E. Archsmith \\ Erich Muehlegger \\ David S. Rapson \\ Working Paper 28933 \\ http://www.nber.org/papers/w28933 \\ NATIONAL BUREAU OF ECONOMIC RESEARCH \\ 1050 Massachusetts Avenue \\ Cambridge, MA 02138 \\ June 2021
}

The authors declare that they have no relevant or material financial interests that relate to the research described in this paper. We thank Joshua Linn and Resources for the Future for providing access to data and computing. We gratefully thank Reid Taylor for excellent research assistance, and the editors of this volume for their helpful comments and suggestions on early versions of the paper. All errors are our own. The views expressed herein are those of the authors and do not necessarily reflect the views of the National Bureau of Economic Research.

NBER working papers are circulated for discussion and comment purposes. They have not been peer-reviewed or been subject to the review by the NBER Board of Directors that accompanies official NBER publications.

(C) 2021 by James E. Archsmith, Erich Muehlegger, and David S. Rapson. All rights reserved. Short sections of text, not to exceed two paragraphs, may be quoted without explicit permission provided that full credit, including (C) notice, is given to the source. 
Future Paths of Electric Vehicle Adoption in the United States: Predictable Determinants, Obstacles and Opportunities

James E. Archsmith, Erich Muehlegger, and David S. Rapson

NBER Working Paper No. 28933

June 2021

JEL No. H23,Q47,Q48,Q5,R4

\begin{abstract}
$\underline{\text { ABSTRACT }}$
This paper identifies and quantifies major determinants of future electric vehicle (EV) demand in order to inform widely-held aspirations for market growth. Our model compares three channels that will affect EV market share in the United States from 2020-2035: intrinsic (no-subsidy) EV demand growth, net-of-subsidy EV cost declines (e.g. batteries), and government subsidies. Geographic variation in preferences for sedans and light trucks highlights the importance of viable EV alternatives to conventional light trucks; belief in climate change is highly correlated with EV adoption patterns; and the first $\$ 500$ billion in cumulative nationwide EV subsidies is associated a 7-10 percent increase in EV market share in 2035, an effect that diminishes as subsidies increase. The rate of intrinsic demand growth dwarfs the impact of demand-side subsidies and battery cost declines, highlighting the importance of non-monetary factors (e.g. charging infrastructure, product quality and/or cultural acceptance) on EV demand.
\end{abstract}

James E. Archsmith

Department of Agricultural

and Resource Economics

2200 Symons Hall

University of Maryland

7998 Regents Drive

College Park, MD 20742-5535

archsmit@umd.edu

Erich Muehlegger

University of California,Davis

Economics Department

One Shields Ave

Davis, CA 95616

and NBER

emuehlegger@ucdavis.edu
David S. Rapson

Department of Economics

University of California, Davis

One Shields Avenue

Davis, CA 95616

dsrapson@ucdavis.edu 


\section{Introduction}

The rate of transportation electrification in the United States will be determined by three forces: intrinsic growth in demand for electric vehicles (EVs), production cost declines, and government stimulus of the industry. Many climate change mitigation plans incorporate rapid adoption of light-duty EVs as a central pillar of decarbonization efforts. The aspiration in many circles is to achieve 100 percent electric transportation and several countries and states have declared their intention to ban gasoline car sales. ${ }^{1}$ To ascertain the feasibility (and even the desirability) of these goals, a clearer understanding of the obstacles and opportunities to widespread EV adoption is needed.

This paper has three main goals. First, we seek to identify cross-sectional patterns in recent adoption of light-duty cars and trucks using a large, nationwide micro-level dataset of car purchases from the market research firm MaritzCX ("Maritz"). These patterns reflect potential determinants of future EV adoption in the US. Second, we demonstrate the important role that growth in intrinsic demand (as opposed to subsidy-induced demand) plays in achieving targets for future EV market share. ${ }^{2}$ For our analysis, "intrinsic demand growth" includes such factors as increased widespread acceptance of EVs, the convenience of charging infrastructure, and the effect of EV quality improvements that will likely result from continued technological advancements in battery production. Third, we use a range of plausible EV demand elasticities to estimate the effect of EV purchase subsidies. This allows us to present the range of aggregate subsidy bills that will be required to meet different EV market share targets by 2035 under a given set of assumptions. The reader may then observe the subsidy bill associated with her preferred assumptions about intrinsic EV demand growth, the demand elasticity of EVs, and the availability of viable substitutes for gasoline-powered light trucks. ${ }^{3}$

Methodologically, this exercise requires developing EV market share forecasts - the range of counterfactual levels of adoption that could be expected to occur without government subsidies. We use Maritz microdata to identify cross-sectional drivers of EV demand using a probit model. This model can be projected forward to include factors such as population and income growth, which are allowed to vary at the state level. We then adjust these forecasts upwards to account for a range of intrinsic growth rates in EV demand. Policy nudges and changing tastes for EVs will have heterogeneous impacts across the population. Through our modeling

\footnotetext{
${ }^{1}$ https://www.coltura.org/world-gasoline-phaseouts

${ }^{2}$ Throughout the paper, "EV" refers to both battery electric (BEV) and plug-in hybrid vehicles (PHEV), and "EV market share" refers to the share of new car sales in the US light duty market.

${ }^{3}$ Throughout, we use the term "light trucks" to include sports utility vehicles (SUVs), minivans and pickup-trucks.
} 
approach price effects and intrinsic demand influence future market shares in ways that make intuitive sense; consumers close to the margin for purchasing an EV are much more responsive to changes in prices or intrinsic demand that consumers far from the margin. This leads to substantial spatial heterogeneity in the impact of subsidies on future EV market shares.

The results of these scenario forecasts reveal several key insights into the future trajectory of EV market shares. First, demographic and geographic differences in vehicle preferences are large and important. EV demand is strongly correlated with higher levels of income and education, and EV adoption is highest among car buyers 35 to 45 years old. More liberal and coastal states tend to have higher demand for sedans and EVs which, to date, are primarily available as sedans. On the other hand, car buyers in interior states have substantially higher demand for light trucks and lower demand for EVs. These patterns speak to the importance of a portfolio of EV light trucks in achieving adoption targets. Since light trucks comprise over half the new cars sold in the U.S. and have not been widely available in EV powertrains, it is difficult to rely on revealed preference approaches to estimate future demand in this crucial market segment. If (or when) a robust slate of EV light trucks is available, the difference between the demographics of EV buyers and those of buyers of conventional light trucks suggest an uphill climb for EV adoption in the interior of the country. These demographic patterns mirror stated environmental preferences - EV demand exhibits a strong, positive correlation with stated preference for environmental cleanliness, and regions with high light truck market share report low rates of concern about climate change.

Second, the rate of growth in intrinsic demand for EVs is the most important determinant of future EV market share. It reflects the extent to which, in the absence of government subsidies, buyers prefer EVs to gasoline-powered cars. Our "high", "medium" and "low" growth scenarios intend to capture the wide range of potential outcomes in the market. Beliefs about the intrinsic growth rate going forward are likely to differ from reader-to-reader. In the absence of government subsidies, and assuming that EV light trucks have emerged as viable substitutes in that segment, the low-growth scenario leads to a nationwide EV market share of 10 percent in 2035. The medium- and high-growth scenarios lead to EV market shares of 17 and 42 percent, respectively. These nationwide market shares obscure substantial geographic heterogeneity. In the medium-growth scenario, EVs have a 2035 market share of 26 percent in California and New Jersey, and 14 to 16 percent in states like Mississippi, Arkansas and Louisiana.

Third, government subsidies can be an effective tool for stimulating EV demand, but the aggregate subsidy bill grows dramatically as the size of the EV market increases. Assuming 
an EV demand elasticity of 2.0, the first $\$ 500$ billion in cumulative EV subsidies over the period 2020-2035 increases EV market share by roughly 7 to 10 percentage points in each of the (low/medium/high) intrinsic growth scenarios. If the reader prefers a higher demand elasticity (3.0) or lower (1.0), the first $\$ 500$ billion in subsidies has a slightly larger (10 to 12 percentage points) or smaller (5 to 6 percentage points) increase, respectively. However, there are diminishing returns. Under the 2.0 elasticity assumption, each subsequent $\$ 500$ billion increases EV market share by closer to 5 to 8 percentage points.

These findings offer several considerations for policymakers. Geographic heterogeneity in the demand for EVs reinforces the efficiency of policies that differ across time and space. ${ }^{4}$ The growth rate in EV market share should be monitored by authorities, and may inform optimal adjustments in EV targets. This is particularly true when considering the optimal level of consumer purchase subsidies for EVs. The subsidy bill required to achieve even moderate EV market share could likely measure in the hundreds of billions of dollars, and possibly trillions, and the effect of these subsidies is dwarfed by other factors that influence the intrinsic demand for electrified transportation services.

Section 2 describes the datasets that we use; Section 3 discusses recent trends and correlations in EV adoption; Section 4 describes the probit model and methodology underlying the intrinsic growth rate scenarios; and Section 5 presents the main results and implications for EV adoption in the US. Section 6 concludes.

\section{Data}

This section describes our three data sources. The primary data source is a large-scale, nationwide survey of vehicle purchase decisions, which we use to investigate cross-sectional patterns in demand. The forecasting scenarios also require data on state-level EV incentives and demographic forecasts.

\subsection{Vehicle choice survey}

The primary data underlying our analysis is a survey of US new vehicle purchasers conducted by Maritz. The survey is sent to a random sample of households who purchased a new vehicle in the past year. Over 200,000 individuals respond each year. The survey includes questions about vehicle attributes, intended uses, personal attitudes which may impact vehicle choice,

\footnotetext{
${ }^{4}$ See Muehlegger and Rapson (2021) for a deeper, complementary discussion of this observation.
} 
preferences over hypothetical vehicle attributes, financing and purchase details, household composition, demographic information, and vehicle holdings, and forward looking questions about future vehicle purchases.

Maritz additionally incorporates information such as the home zip code of the buyer and portions of the Vehicle Identification Number (VIN) that are known at the time of vehicle purchase. We match supplied components of the VIN with a VIN decoder from DataONE to determine detailed vehicle attributes such as the powertrain type and - in the case of EVs - the battery capacity and all-electric range.

We rely on data for vehicles purchased in the 2017 and 2018 calendar years, which are the most recent years available at the time of this writing. This is a rich source of information on electric vehicle purchases. Of the 474,274 survey responses during this period, 6,196 were purchases of BEVs and a further 9,280 were PHEVs covering the full range of BEV and PHEV models available at the time.

While surveys are sent to a random sample of new vehicle purchasers, responses are a nonrandom subset of those responses. In all analyses, we re-weight the responses using nationally representative sampling weights provided by Maritz. These weights balance the composition of vehicle make-model pairs in survey responses with the true national distribution.

\subsection{Incentives}

To calculate the subsidy available to the buyer, we focus on large-scale, state-wide incentive programs which provide monetary subsidies for electric vehicle purchases, either through rebates or tax-credits, as well as the federal tax credit. We do not account for corporate incentive programs, such as those provided locally by utility companies, local government subsidies, or charging infrastructure incentives. We compile data on the amount of subsidy, the qualification requirements, and eligible purchase dates for each program primarily from the database of laws and incentives from the Alternative Fuels Data Center (AFDC) at the US Department of Energy. ${ }^{5}$ The AFDC database is updated at the closure of each state's legislative season, and contains information on current and expired subsidy programs. Supplementary information is sourced from state specific program websites, state departments of revenue websites, automotive industry websites, and contemporary news articles for expired programs. For the years of the sample, 2017-2018, sixteen states operated incentive programs. Table 1 details the programs, dates, and the maximum subsidy offered by each state. As we observe purchase

\footnotetext{
${ }^{5}$ https://afdc.energy.gov/laws
} 
date, income, and vehicle characteristics, we are able to accurately attribute potential subsidy amounts, which vary along these dimensions in most states. However, we do not observe actual subsidy take-up at the individual level. Conditional on qualifying for a state-level subsidy, the average subsidy amount is $\$ 2,164$ with 46 percent of nationwide EV sales qualifying for a state level subsidy.

\subsection{Demographic forecasts}

Demographic forecasts account for changes in population by age group and race across states. We collect forecast data from the Demographics Research Group at the University of Virginia's Weldon Cooper Center. Population estimates are published for 2020, 2030, and 2040, broken out by state, race, age group cells. The model is built upon data from the past three decennial national census from the U.S. Census Bureau as well as the July 2017 population estimate update from the Bureau. 2020 state population totals are projected from 2010 and 2017 state levels, assuming an exponential growth pattern. 2030 and 2040 estimates use the Hamilton-Perry projection approach and account for birthrate, mortality, and migration patterns. ${ }^{6}$

\section{Past Evidence on EV Adoption}

The goal of the this paper is to project EV market share to (1) understand the feasibility and costs associated with achieving ambitious fleet adoption targets, and (2) compare the relative importance of various drivers of EV adoption. But before we discuss the projection of EV market share, we summarize historical trends in rates of adoption and the cross-sectional variation relevant to our analysis.

Early adoption of EVs, like early adoption of many other new technologies, has experienced dramatic growth in a short period of time. Figure 1 graphs the 3-year moving average yearon-year growth rate of EV sales from 2012 to the present for California and the U.S. more generally. In the first few years of EV sales, sales doubled every year or two. Yet, similar to many other new technologies, as annual sales increase, year-on-year growth rates decline reflecting both the greater difficulty in achieving a high growth rate over a larger base of sales and the maturation of the technology. In the case of EVs, the year-on-year growth rate has fallen to roughly 30 percent for the U.S. (and slightly lower for California where the market

\footnotetext{
${ }^{6} \mathrm{~A}$ detailed overview of the population forecasting methods can be found here: https://demographics. coopercenter.org/sites/demographics/files/2019-02/NationalProjections_MethodologyDverview_Dec2018. pdf
} 
share of EVs is higher).

The growth rates illustrated in Figure 1 represent adoption that, up to the present, has been selective in many ways. As an example, California's robust rates of EV adoption have been supported by aggressive state-level incentives, supplementary to those offered federally, with the goal of further closing the upfront cost gap between electric and conventional vehicles. In addition, the population of California might be predisposed to adopt EVs, even in the absence of a subsidy. The population is relatively wealthy, liberal, and concerned about climate change. Drivers in the state have a relative preference for sedans for which a suite of electric-powered options are available, which draws a distinction with light trucks for which current EV options are limited. Rates of adoption have lagged in states with a higher rural population share, states that offer little in the way of supplementary EV incentives, or states with populations less engaged with environmental issues and more likely to purchase light trucks .

Regional purchase patterns in the Maritz data highlight the correlation between state-level EV adoption and preferences for different types of vehicles. Figure 2 maps the market shares of EVs, sedans, SUVs and pickup trucks by state, as reported in the Maritz data. As the top two panels illustrate, states that have experienced relatively high EV adoption to date (top-left panel) were states that also had a relative preference for sedans. In contrast, many of the states with relatively low EV adoption to-date are states whose drivers reveal a preference for vehicle classes currently lacking models with electric powertrains. The central challenge in our paper is unpacking the various drivers of EV adoption so as to understand whether adoption rates in much of the country will catch up to those in California or whether adoption in many parts of the country will continue to lag, even after EV prices fall closer to parity with conventional vehicles and automakers expand the suite of EV light trucks.

As a starting point for examining cross-sectional variation in adoption, we summarize the demographics of EV purchasers in the Maritz data to illustrate the selection into the decision to purchase an EV. The graphs in Figure 3 report average EV adoption by income, age education and ethnicity. The relationship between early EV adoption and income aligns with previous academic evidence (e.g., Borenstein and Davis (2016)) which finds evidence that early tax credits accrue disproportionately to high income households. From a policy perspective, the strong correlation between adoption and incomes motivates policymakers to means-test EV incentives, so as to better achieve distributional objectives (see e.g., the EFMP program studied by Muehlegger and Rapson (2018) and adjustments to California's Clean Vehicle Rebate Program in and after 2016). Although our data cover a later time period than that covered by Borenstein 
and Davis (2016), we also observe that EV ownership and incomes are positively correlated, reflective of the high cost of upfront purchase and the continued relative scarcity of used EVs. Less well documented are the relationships between other sociodemograhic variables and EV adoption. Educational attainment, like income, is strongly correlated with EV adoption. College graduates and those with post-graduate degrees are roughly three times as likely to own an EV as less educated drivers. In the bottom two panels, patterns of ownership emerge for age brackets and self-designated ethnicity - middle age drivers (between ages 35 and 44) are the most like to own EVs, as are Asian-Americans.

Just as demographics vary for buyers of electric vehicles and conventional light trucks, so do the environmental attitudes of areas preferring one or the other. In Figure 4 we plot the fraction of the state population that affirmed a belief in climate change (Howe et al. (2015)) against the EV share of the sedan market (left-hand panel) and the light truck share of the overall market. Although we can only speculate as to how current buyers of light trucks might choose amongst electric and conventional options, the left- and right-hand panels of the figure provide suggestive evidence that, even with a suite of EV light trucks, adoption may lag in states that have historically preferred light trucks to sedans. The data in the left-hand panel illustrates a strong positive relationship between environmental beliefs and EV share of the sedan market. While some of the positive relationship is likely driven by the endogeneity of state-level incentives (such as the largest circle, representing California), state-level incentives are only offered by a small subset of states. In contrast, the right-hand panel suggests both substantial heterogeneity in preferences for light trucks, but also a strong negative relationship between environmental beliefs and light truck market share. Not only are states with a more environmentally concerned population more likely to adopt EVs, they also have a relative preference for the vehicle classes for which EVs have been most available to date. In contrast, states with a high fraction of light trucks are less likely to purchase EVs conditional on buying a sedan for which EV options are available. To the extent that environmental preferences continue to play a role in consumer decisions to purchase EVs, this suggests that adoption might continue to lag in many parts of the country even after robust EV alternatives are created for market segments outside of sedans.

A final insight follows from comparing the prices of sedans and light-trucks. Although battery prices have declined substantially over the past decade, EVs continue to have higher upfront costs than conventional vehicles. ${ }^{7}$ While state and federal subsidies have reduced

\footnotetext{
${ }^{7}$ As one example, the MSRP of an entry-level 2021 Nissan Leaf EV is $\$ 31,670$ (before incentives) while the starting price of its gasoline counterpart, the 2021 Nissan Versa, is roughly $\$ 15,000$.
} 
or, in rare cases, eliminated this upfront price differential, EV light trucks likely face greater price disadvantages due to higher battery capacity requirements. ${ }^{8}$ Larger batteries are more expensive than smaller ones. Moreover, the underlying price of light trucks tends to be greater than those of sedans as illustrated by the histograms of manufacturer suggested retail price (MSRP) for conventional sedans and light trucks in the left- and right-hand panels of Figure 5. Collectively, this suggests that the state and federal subsidies at currently levels will have a more modest impact in percentage terms on the upfront price of an electric light truck.

\section{Modeling Future Scenarios}

Our primary goal is to forecast vehicle purchase behavior under various estimates of and assumptions about vehicle class preferences, technological advancements and cost declines. To do so, we combine cross-sectional evidence on the characteristics of past buyers of EVs and past buyers of sedans with a projection of the continued rate of growth in intrinsic demand for EVs.

\subsection{Estimating cross-sectional patterns in adoption}

Using the individual-level Maritz survey data of recent new vehicle purchases, we model a discrete purchase choice of individual $i$, such as whether the vehicle is classified as a light truck or is an EV, as a function of the purchased vehicle price $\left(p_{i}\right)$, a vector of demographic information of the purchaser $\left(X_{i}\right)$, and an unobserved error $\mu_{i}$. We additionally include a term representing consumers' intrinsic preference for the attribute $\left(\delta_{i}\right)$, which can change over time. It is assumed to be zero during the during the period used to estimate model parameters, but may change in the future as consumer preferences change. We assume the market share of choice $y$ is the inverse normal with variance one of a linear combination of these factors. In this probit framework, the probability that consumer $i$ chooses a vehicle with discrete attribute $y$, and hence the market share of $y(S(y))$, is

$$
S(y)=\operatorname{Pr}\left(Y_{i}=y\right)=\Phi\left(\alpha p_{i}+X_{i} \beta+\delta_{i}+\mu_{i}\right)
$$

where $\Phi(\cdot)$ is the cumulative distribution function of the standard normal.

Paramount among these drivers of consumer choice is vehicle price. Rather than em-

\footnotetext{
${ }^{8}$ As a point of reference, the 2021 Nissan Leaf, Tesla Model 3 and Tesla Cybertruck currently list battery capacities of $40-62 \mathrm{kWh}, 50-82$, and $100-200 \mathrm{kWh}$, respectively.
} 
pirically estimate the impact of prices on purchase decisions, we rely on estimates of priceresponsiveness from the literature. We assume the price elasticity of new vehicle demand is -0.4. We additionally model substitution between conventional cars and EVs and assume, conditional on purchasing a vehicle of a given class, the elasticity of demand for EVs is -2.0. We have chosen -2.0 as an approximate midpoint of the range of existing estimates that aren't focused exclusively on lower-income segments (which are likely to be more price elastic). ${ }^{9}$ All else equal, a higher demand elasticity increases the effectiveness of subsidies.

In the probit formulation above, the price elasticity of market share will vary across the space of observed prices and demographic attributes. We choose a value of $\alpha$ so this elasticity is equal to the targeted value at the mean market share. Formally, if $S(y)$ is the market share of attribute $y$ and $\bar{p}$ is the average price of a good with that attribute, the price elasticity of market share is

$$
\frac{\partial S}{\partial \bar{p}} \frac{\bar{p}}{S}=\alpha \phi\left(\Phi^{-1}(S)\right) \frac{\bar{p}}{S}
$$

Solving for $\alpha$ we find the value of the price parameter that leads to the desired price elasticity (e) of market share at the mean:

$$
\alpha^{*}=\frac{e \cdot S}{\phi\left(\Phi^{-1}(S)\right) \cdot \bar{p}}
$$

We then estimate the remaining model parameters $(\beta$ and $z$ ) from observed purchase behavior using the probit, including the price effect $\alpha^{*} \cdot p$ as a fixed value with its coefficient constrained to one.

We estimate two functions mapping demographics to market shares. First, amongst all vehicle purchases, our "Vehicle Class" market share model $\left(S^{T}(\cdot)\right)$ estimates the relationship between demographics and the market share of vehicles classified as "light trucks" (i.e., an SUV, pickup truck or van) as $Y^{T}=1$ or vehicles we will classify as "cars" (i.e., a coupe, sedan, or hatchback) as $Y^{T}=0$. Second, amongst only purchases of cars, we estimate the relationship between demographics and the market share of EVs (i.e., a BEV or PHEV) as $Y^{E V}=1$ or a vehicle with some other powertrain as $Y^{E V}=0$ in the "Powertrain" model $\left(S^{E V}(\cdot)\right)$.

Combining these models, allows us to predict the probability a consumer with a given set

\footnotetext{
${ }^{9} \mathrm{Li}$ et al. (2017) uses gasoline prices as an IV and estimates a demand elasticity of -1.3. Springel (2017) and Li (2017) both use BLP IVs to retrieve estimates of -1.0 to -1.5 (Springel) and -2.7 (Li), respectively. Muehlegger and Rapson (2018) use variation from a low- and middle-income EV subsidy program to estimate an elasticity of -3.3 in that subsegment. We also present alternative scenarios with elasticities ranging from -1.0 to -3.0 and heterogeneous elasticities by income.
} 
of demographic variables $\left(X_{i}\right)$ would purchase a given vehicle - such as an EV car - as outlined in Figure 6. We first apply the Vehicle Class model to determine the probability a consumer would elect to purchase a vehicle in the car class, and then apply the Powertrain model to determine the likelihood, conditional on purchasing a vehicle, they elect to purchase one with an EV powertrain. ${ }^{10}$

\subsection{Future EV Adoption Scenarios}

Based on the estimates from current patterns of adoption, we examine several scenarios to understand how EV entry into the light duty truck segment and government subsidies might play a role in achieving national adoption targets. Equipped with estimates of the vehicle class and powertrain models, we are able to generate counterfactual probabilities of vehicle purchase for hypothetical consumers across the space of possible combinations of demographic variables. To simulate future vehicle purchases, we construct a population of individuals with demographic attributes matching the expected distribution of those attributes in the future, generate predicted purchase probabilities for each individual, and then aggregate across the entire population.

Historical trends and forecasts can allow us to predict the distribution of some of these demographic variables into the future (e.g., household income, population-by-age). However, many of the demographic attributes entering into these models are not forecasted (e.g., the number of future households that will value a vehicle for its towing capacity). Further, knowledge of the joint distribution of these attributes is critical for constructing a hypothetical future population. Rather than attempt to construct this joint distribution from the ground-up, we take a top-down approach starting with the current distribution of demographic attributes as observed in our data to construct the future joint distribution of demographic attributes. To do so, we assume real household income increases at 1.2 percent per year, the average annual rate of income growth in the United States between 1970 and 2018. Household incomes increase over time without altering other demographic attributes. Second, we account for changes in population by reweighing the distribution of demographic variables so that it matches the distribution of population in state by 5-year age bins in forecasts from Demographics Research Group (2018). ${ }^{11}$

\footnotetext{
${ }^{10}$ To be clear, the lack of EV light trucks to-date prevents us from estimating the probability with which a lighttruck buyer would purchase an EV light truck from revealed preference data. Thus, as outlined in Figure 6, we use the observable data on EV sedan purchases as a guide for the conditional probability that a future light truck buyer chooses to purchase an model with an electric powertrain.

${ }^{11}$ Demographics Research Group (2018) forecast population in each state and 5-year age bin at 10 year intervals. We
} 
Specific to the market for EVs, we focus on three factors that shift the future path of EV adoption. The first of these relates to the continued growth rate of intrinsic EV demand. One may expect that as the quality, range, or set of EV available models increases consumers may be more likely to purchase an EV, regardless of the price. Further, charging infrastructure density and range limitation perceptions may continue to evolve over time in ways that increase demand for EVs. The intrinsic growth rate reflects the subsidy-free rate of growth in EV adoption that may result from these and other non-price factors. These may include improvements in EV technology, build-outs in EV charging infrastructure, supply-side mandates, extensions of the product suite, and any network externalities that may result from EVs becoming a greater fraction of the vehicle fleet.

The intrinsic growth parameter is one of the most glaring unknowns facing the vehicle industry, policymakers and researchers. Figure 1 shows the three-year moving average annual growth rate in EV sales for California (orange/light) and the rest of the United States (black/dark). To date, year-over-year growth in sales has generally exceeded 20 percent per year but has been declining in a manner similar to the growth rates for many other new technologies. This is to be expected in a young and maturing industry. In our analysis, we calibrate year-over-year demand growth in 2020 to the observed value of 30 percent. Acknowledging demand for EVs cannot grow at this rate indefinitely, we assume this growth rate decreases over time. We construct three benchmark scenarios wherein the year-on-year rate of growth of the intrinsic demand for EVs declines by 5 percent (high growth), 10 percent (medium growth) or 15 percent (low growth) per annum.

Under these scenarios, intrinsic demand for (and the resulting market share of) EVs will initially grow quickly, but at a rate that decreases and approaches zero over time. Figure 7 shows how these scenarios predict EV market shares will evolve over time, holding all other factors constant. Panel (a) shows EV market shares through our forecast horizon of 2035. Since growth of EV market share is compounding, it is clear that small changes in the assumed rate of EV market share growth have large impacts on market shares in 2035. Panel (b) demonstrates the difficulty in using past data to predict future market share growth. Starting in 2020, we backcast market shares from each of these scenarios to 2011 and plot them along actual EV market shares. Each of the high, medium and low growth rate scenarios is consistent with the history of observed market shares. Finally, Panel (c) shows the assumed year-over-year growth rates for each scenario in each year through 2035. Under the high growth scenario, EV

interpolate forecast population in the intervening years using a constant growth rate. 
market shares are assumed to be increasing at nearly 15 percent per year in 2035 and at around 4 percent per year under the low growth scenario.

We allow for the role of these time-varying preferences for EVs to enter into our market shares through the intrinsic preference parameter $\delta_{i}$. This parameter is assumed to be zero during and before 2020, but is allowed to change in future years as consumer preferences for EVs change. We compute the value of this intrinsic preference parameter as the value $\delta_{i}^{*}$ that rationalizes the assumed growth in EV market shares under a business as usual (no subsidies or demographic change) scenario. We then use this value of $\delta_{i}^{*}$ as the value of intrinsic preference when forecasting EV market shares.

The second factor is continued reductions in the price of EVs, driven either by falling production costs (e.g., as a result of economies of scale in battery production) or through continued or new government subsidies. Recall that we pre-condition the impact of prices out of decisions and demographic attributes prior to estimating the purchase models. After computing predictions from these models, we reincorporate price effects based on the composition of future prices. We assume consumers respond only to the net price of a vehicle (i.e., inclusive of any subsidies) and present several scenarios for the future evolution of EV prices. In all scenarios, we assume prices of non-EVs are constant in real terms.

As a starting point, we assume that the sedan and light truck EV markets reach price parity with gasoline cars in 2030 and 2040 respectively. ${ }^{12}$ At that point, the average (unsubsidized) price of EVs will equal that of gasoline counterparts. In the intervening years, the EV price premium relative to the compatible ICE vehicle falls at a constant rate. The decline in EV prices primarily reflect falling battery costs. This also underlies the rationale for light trucks to reach price parity later, since battery capacity requirements are much higher for these vehicles. Additionally, we consider the impact of an extension of the federal subsidy of $\$ 7,500$ per vehicle. ${ }^{13}$

Finally, we assume that, over time, the EV offerings available in the light truck market expand to saturate the product space. Although it is not possible to forecast the evolutionary path (i.e., range, power and other attributes) of EV light trucks, we make the assumption that by 2035 EV light trucks have reached "quality-parity" with EV sedans, such that the conditional probability of a buyer of choosing an EV conditional on purchasing a light truck is equal to the conditional probability of the same buyer of purchasing an EV when purchasing a sedan.

\footnotetext{
${ }^{12}$ Larger mass and increased aerodynamic drag increase the battery capacity required to achieve a given range in EV trucks relative to EV cars. As batteries represent the bulk of the increased cost of EVs, we assume EV trucks will take longer to achieve cost parity than cars.

${ }^{13}$ We also consider a range of alternative tax-inclusive prices in Table 4.
} 


\section{Results \& Discussion}

\subsection{Factors correlated with EV adoption}

We employ a single set of demographic variables to estimate both the Vehicle Class and Powertrain market share models. Our selection of candidate variables was driven by two factors. First, no question in the Martiz survey requires a response, so we considered only demographic factors that were supplied by at least $80 \%$ of survey respondents. Second, our methodology in forecasting future purchase behavior allows for common demographic factors to change over time. The survey includes an array of questions on the underlying reasons for purchasing a vehicle (e.g., did the consumer purchase the vehicle because they value fuel economy). While these attitudes are likely strong drivers of purchase behavior, there are scant projections of how these attitudes may convolve with other demographic factors over time. Consequently, we exclude responses designed to elicit attitudes underlying purchase reasons. Since we allow demographic factors to enter into these models flexibly, the effect of evolving attitudes will be reflected in forward forecasts to the extent attitudes are correlated with demographic factors included in our model.

The demographic factors included in the purchase model are annual VMT, annual household income, family size, portion of vehicles owned by the household falling into each of four classes (cars, pickups, SUVs, and vans) ${ }^{14}$, age of the primary driver, income of the primary driver, and race of the primary driver. The true relationship underlying our market share models may be nonlinear or depend on interactions of these variables. We consider a space of candidate models of up to third-order interactions of all these candidate variables and select preferred models as the specification that minimizes mean square out-of-sample prediction error using 10-fold cross validation. Using this procedure, the full set of these demographic factors and their second order interactions form the controls in the Vehicle Class model and the full set of demographic factors absent interactions form the set of demographic variables in the Powertrain model. ${ }^{15}$

We evaluate the predictions of these models by forecasting purchase probabilities and comparing to realized purchase behavior. Histograms from out-of-sample predicted probabilities

\footnotetext{
${ }^{14}$ These shares mechanically sum to one so the portion of vehicles classified as vans are treated as the excluded category in estimation.

${ }^{15}$ We performed this cross validation procedure using three empirical models: the standard probit, a regularized probit using a lasso penalty selected via cross-validation, and a post-regularized probit using the set of variables selected by the regularized probit without the lasso penalty. Each approach preferred the same specification for each model. The standard probit produced the lowest out-of-sample forecast error and is the basis of our forecasts.
} 
from each model, grouped by the actual purchase behavior, are shown in Figure 8. The vehicle class model strongly predicts observed behavior. Very few individuals with low predicted probabilities of purchase a truck actually do so. Likewise, few individuals with large predicted probabilities of purchasing a truck actually purchase a car instead. The powertrain model is not as strongly discriminating between EV and ICE purchases, revealing the extent to which unobservable factors drive the EV adoption decision. However, the model still clearly has predictive power, with a substantially higher density predicting EV purchases amongst the population of consumers who actually purchased EVs.

\subsection{Future EV adoption paths}

The primary goal of our paper is focus attention on three important drivers of future EV adoption: (1) assumptions about the intrinsic (subsidy-free) rate of growth of EV sales, (2) the role of subsidies or further production efficiency gains that push EVs below the point of cost-parity with conventional vehicles, and (3) the importance of a robust portfolio of electric light trucks that complement the slate of electric sedans.

As discussed above, we model future adoption by combining the cross-sectional variation from the Maritz data (reflecting preferences for EVs and preferences for light trucks), with a projection of how intrinsic preferences has changed since the inception of the industry. Table 2 presents six panels, each of which presents the market share of EV sedans, EV trucks and the totals for the market through 2035 for one of the considered scenarios. Each row of scenarios correspond to one of the three assumptions about the future rate of intrinsic growth rate in the demand for EVs. ${ }^{16}$ The left column of scenarios assumes that EVs reach price parity in 2030 and remain there. The right column of scenarios assumes, in addition to price parity, a continuation of the federal subsidy of $\$ 7,500$ per vehicle. Although we frame these scenarios as a continuation of the federal subsidy, since the consumer only cares about the subsidy-inclusive price of the vehicle, the right column of scenarios could also be thought of arising from productivity gains that push the sticker price of $\mathrm{EVs} \$ 7,500$ below price parity with conventional vehicles.

Table 2 illustrates the relative importance of the three drivers of future EV adoption. Perhaps unsurprisingly, the most important of the three factors is the rate at which intrinsic growth declines going forward. As illustrated in Figure 7, even a relatively modest change in the

\footnotetext{
${ }^{16}$ As stated above, in all six scenarios we assume that the intrinsic (i.e., subsidy free) growth rate starts at 30 percent per annum in 2020 . The rate then declines by 5, 10 or 15 percent per annum thereafter, in the high, medium and low growth scenarios.
} 
growth of the intrinsic growth rate leads to very significant differences in the rates of future EV growth. In the "high" scenario, where intrinsic rates of EV adoption decline by only 5 percent per year, the growth rate declines slowly from approx 30 percent in 2020 to roughly 15 percent per year in 2035, whereas in the "low" scenario, the per-year growth rate in EV adoption quickly dips into the single digits. These differences translate into large differences in adoption in panels (a), (c) and (e). In the more optimistic of the scenarios, EV market share rises to over 40 percent, split roughly equally between EV sedans and EV light trucks, by 2035 . In the least optimistic case, in which the growth rate falls to single digits later this decade, the market share of EVs stays below ten percent through 2035. Figure 9 provides a graphical illustration of the evolution of predicted market shares over time.

Although we remain agnostic as to which of the three assumptions about intrinsic growth rates is the most appropriate, the data suggest that the intrinsic growth rate is both central to any projection and likely difficult to estimate from historical data. Notably, when "backcasting" the historical market share of EVs in Figure 7, panel (b), all three growth rate scenarios do a reasonable job. This suggests that, although the rate of intrinsic growth is a very important driver of the future adoption path, historical data does not provide a clear guide as to how intrinsic growth might evolve of the next two decades.

Next, we consider the impacts of a continuation of the $\$ 7,500$ per vehicle federal subsidy. The left-hand and right-hand columns of scenarios in Table 2 contain the projections without and with the federal subsidy, respectively. ${ }^{17}$ Relative to the impact of the intrinsic growth rate, the impact of subsidies is more modest, although still considerable. Across the low, medium and high growth rate scenarios, subsidies increase the market share of new vehicles by three to six percentage points, split roughly equally between the EV sedans and EV trucks. The smaller impact of subsidies reflects the challenge of expanding adoption into populations and locations that have generally been less receptive to EVs to date.

It is important to note that the projections implicitly assume that the subsidies accrue entirely to the buyers of EVs. Although Muehlegger and Rapson (2018) finds evidence of rates of pass-through close to one for the Enhanced Fleet Modernization Program, a pilot program targeted as low and middle-income households in California, no paper empirically estimates the pass-through of the federal tax credit on EVs. If the pass-through rate declines as the program expands in size, we would expect more modest impacts of subsidies on adoption.

\footnotetext{
${ }^{17}$ Alternatively, as the consumer doesn't distinguish between a subsidy and a reduction in the subsidy-exclusive sticker price, the right-hand column can be interpreted as a scenario in which efficiency gains lead the sticker price of EVs to be well below price parity with comparable ICE vehicles by 2035.
} 
Finally, we turn to the importance of a viable, competitive slate of EV light trucks. Light trucks (including pickup trucks, SUVs and vans) are the most popular vehicle class in the United States. Yet, electrification of the light truck segment is still at a nascent stage. In each of the panels, the middle two columns present the market shares of electric sedans and light trucks. Although market shares of electric light trucks are initially low (reflecting the early stage of development), the market share of EV light trucks rises. In 2035, the date at which we assume EV light trucks reach "quality-parity" with EV sedans, the market share of EV light trucks is roughly level with the market share of EV sedans. Mathematically, expansion into the light truck segment offers the potential to increase the overall market shares of EVs considerably, an outcome unlikely to be accomplished through EV sedan sales alone. ${ }^{18}$

The roughly equivalent market shares of EV light trucks and EV sedans by 2035 reflects two competing forces. Light trucks, as a class, are more popular than sedans. As illustrated in Figure 2, panel (b), the light trucks command the majority of the new vehicle sales in virtually every state in the U.S. ${ }^{19}$. Thus, the overall potential for EV light trucks in greater than that for EV sedans, barring a major shift in consumer preferences. But the higher market share of light trucks is tempered by the characteristics of the truck buyers, who tend to have less strong preferences for EVs, all else equal, than sedan buyers (see Figures $4 \mathrm{a}$ and $4 \mathrm{~b}$ ).

\subsection{Implications for achieving national EV goals}

As an alternative way of evaluating the feasibility of reaching different adoption targets, we calculate the necessary reductions in the subsidy-inclusive price of EVs, and the implied cumulative government subsidy bill, required in each of our scenarios. Again, despite our framing of the "subsidy effect" that will follow, our model does not distinguish between the role of subsidies and the role of falling production costs. From the perspective of the consumer purchasing an EV, a dollar of savings arising from lower production costs is equivalent to a dollar of savings arising from more generous government support received at the time of purchase. Thus, we focus on how much the subsidy-inclusive price must decline to meet different adoption targets in our model. Whether these price reductions are feasible in a "no-subsidy" world is left to the reader's judgment.

\footnotetext{
${ }^{18}$ If potential buyers of EV light trucks would have purchased a conventional light truck in the absence of an electric option, EV market share would fall by the entire market share of EV light trucks in a world in which they were not available. If some of these buyers would purchase an EV sedan instead, though, the lack of EV light trucks would have a more modest effect on EV market share.

${ }^{19}$ Collectively, trucks, SUVs, crossovers, and vans command over 70 percent of the U.S. new car market. https://www.nada.org/nadamarketbeat/
} 
A consensus does not exist about the desirable EV market share in the future, or the expected share. California has stated an intention to achieve 100 percent EV market share by 2035. Under the International Energy Agency's Sustainable Development Scenario, the worldwide market share of EVs will need to reach 30 percent by 2030 (International Energy Agency (2020)). Milovanoff et al. (2020) estimate that EVs must comprise 90 percent of the vehicle US vehicle fleet by 2050 in order to limit warming to under two degrees Celsius. Private sector predictions range from 50 percent EV market share in 2030 (UBS), roughly 50 percent in 2035 (BNEF), and 18 percent in 2030 (IHS Markit).

To frame the discussion, we focus on the subsidy requirements to achieve 20 and 35 percent nationwide US market share in 2035 under our low, medium and high intrinsic growth rate scenarios. These targets represent a substantial increase from the present EV market share in the US. Higher targets, while potentially desirable, are either infeasible or prohibitively expensive under our low and medium intrinsic growth rate scenarios.

Figures 10 and 11 show the relationship between EV market share in 2035 and the required level of government subsidies, where the latter are reported both in per-car terms (Figure 10) and as the cumulative subsidy bill required from 2020 to 2035 (11). ${ }^{20}$ We estimate that, to achieve a 20 percent EV market share in 2035, the cumulative subsidy bill under the low and medium intrinsic growth scenarios will be $\$ 710$ and $\$ 140$ billion, respectively. To achieve 35 percent EV market share in 2035, the cumulative subsidy bill increases to $\$ 2.7$ and $\$ 1.3$ trillion, respectively, in the low and medium growth scenarios. If high intrinsic growth is achieved, the 2035 market share will exceed 40 percent in the absence of subsidies, so no subsidies would be required.

Achieving the higher level of EV market share of 50 percent in 2035 would require dramatically higher subsidies, all else equal. In the low growth scenario it would be practically infeasible, requiring subsidies in excess of $\$ 30,000$ per car. Intrinsic demand in the medium growth scenario would need to be augmented by $\$ 3.6$ trillion in total subsidies in order to achieve 50 percent market share in 2035, a figure that falls to roughly $\$ 480$ billion if high intrinsic growth is achieved.

In summary, the cost of reaching ambitious EV market share targets may easily reach trillions of public dollars. These subsidy estimates reinforce one of the main conclusions of this paper: that, while it is possible for subsidies to increase the market share of EVs, subsidies are expensive and have less impact on the rate of EV adoption than other factors that influence

\footnotetext{
${ }^{20}$ The cumulative bill is expressed as a present value in 2020 dollars, and future expenditures are discounted at a 3 percent discount rate.
} 
intrinsic growth in demand. One such factor may be the density of charging infrastructure. Indeed, some papers have argued that a dollar spent on charging infrastructure will induce more EV demand than a dollar spent on consumer purchase subsidies (e.g. Li et al. (2017)). The availability of EV light trucks is likely to be even more important, and has the benefit of being a feature that the market is likely to provide even in the absence of government support.

\subsection{Implications for sub-national adoption}

Although the focus of this paper is on national adoption trends, we examine whether EV market share rises evenly across all states or whether some states (e.g., California) remain persistently ahead, even after light-truck EVs are well-established. As we are not including any state-level incentives, patterns of EV adoption speak to the importance of the underlying preferences of vehicle buyers. In essence, we ask whether, once a slate of attractive, electric light trucks exists, will adoption patterns in much of the interior of the U.S. rise to meet adoption patterns on the East and West Coasts? Or will adoption in the interior of the U.S. continue to lag behind, due to the mix of potential buyers?

In Figure 12, we overlay the projected market share of EVs (in total), electric sedans and electric light trucks on maps of the U.S. in panels (a), (b) and (c) respectively. Panel (a) illustrates the substantial variation in adoption across states, even after a competitive slate of light-truck EVs exists. Although market shares have risen in all states, the market share of EVs remains much higher on the coasts than in the interior of the country, reflective that buyer demographics are more "EV-friendly" on the coasts than in the interior.

From the perspective of environmental policy, relatively lower rates of adoption in the upper Midwest are beneficial, at least in a future world in which the marginal unit of the electricity grid in the Upper Midwest remains more carbon-intensive than the marginal unit of electricity on the coasts. Holland et al. (2016) documents that EVs are generally beneficial from an environmental perspective on the West Coast, due to low carbon-intensity sources of electricity. In contrast, EVs are significantly less beneficial relative to a comparable conventional vehicle in many other parts of the country, although as Holland et al. (2020) notes, the gap between the midwest and other parts of the country have been closing over the last decade. Yet, uneven rates of adoption also raise potential concerns of environmental equality, if rates of EV adoption are significantly higher in more affluent states and cities.

Separating sedan and light-truck EV sales further reveals why adoption rates vary substantially across states. In panel (b), state-level variation in market share of EV sedans is similar 
to the variation that exists today. States with buyers who have a relative preference for sedans and are more "EV-friendly" (e.g., California) have higher rates of adoption than states with buyers who prefer trucks. In Panel (c), which maps the market share of EV light trucks in 2035, we see much more heterogeneity. Particularly instructive is the comparison between Colorado and Wyoming. Both Colorado and Wyoming have populations that prefer light-trucks, yet Colorado is higher income and more progressive than Wyoming and, hence, has a population that is more receptive to EV light trucks.

Figures 13 and 14 both suggest that the impacts of the intrinsic growth rate and federal subsidies tend to spread the distribution of market shares rather than compress it. Figure 13 counts of states by different values of EV market share in 2035, under the high, medium and low adoption scenarios. In each panel, the blue and red histograms illustrate the distribution with and without electric light trucks, respectively. High growth rates, mechanically, pull states with historically high adoption away from states with lower rates of current adoption, at least through 2035. Similarly, Figure 14 suggests that subsidies act in a similar fashion. Since sedans tend to have slightly lower costs than light-trucks, a $\$ 7,500$ subsidy is a higher fraction of the overall purchase price for a sedan. Hence, we see slightly higher rates of subsidy-induced adoption along the East and West coasts.

The differential impact of the expansion of electric powertrains into light truck segment is mapped in figure 12. Panel (a) and (b) illustrate the market shares of EVs with and without expansion into light truck segment, while panel (c) maps the difference between the market shares in (a) and (b). Although for all states, adoption increases with the expansion of EVs into the light truck segment of the market, the impact is greatest in states with a historical preference for vehicles types other than sedans.

\subsection{Other Considerations}

\subsubsection{Demand elasticities}

The demand elasticity determines how sensitive the quantity of EVs demanded is to changes in consumer EV prices. The forecasting scenarios described above assume an elasticity in the range of 1.0 to 3.0. This range is informed primarily by the recent literature that estimates EV demand (as described in section 4). For simplicity, the scenarios that we present assume an elasticity that is constant over time and (with the exception of Figure 15 (d)) across the population. The reality is likely to be more complicated.

Future demand could be less elastic than current demand as EVs become a broader category 
in the market for goods (Berry et al. (1995)). Also, differences in demand are likely between market segments. Early adopters of cars have traditionally been thought to be more price elastic (Goldberg (1995)), although one wonders whether this is true of wealthier EV early-adopters (Borenstein and Davis (2016)). The elasticity of less expensive cars is typically higher than that of more expensive cars (Goldberg (1998)), allowing the possibility that EV cost declines will lead to an increase in price elasticity.

To the extent that EV subsidies are means tested, as they are currently in California under the Clean Vehicle Rebate Project, there will be theoretically ambiguous effects on the overall price elasticity. On one hand, means testing will reduce "free-riding" by directing subsidies away from the subpopulation for which subsidies are inframarginal. This is consistent with Muehlegger and Rapson (2018), who find that low- and middle-income have relatively elastic demand for EVs. All else equal, this would cause means testing to increase subsidy costeffectiveness. On the other hand, if latent demand for EVs is higher among wealthier households, targeting subsidies exclusively towards lower-income households may induce only a modest increase in demand. This would be likely to the extent low-income households perceive low-cost gasoline cars as preferable to subsidized EVs.

Other factors may also affect the EV elasticity, such as the demand for used EVs in a functioning secondary market. Chevalier and Goolsbee (2009) present evidence of forward-looking buyers in primary durable goods markets, where demand for the new durable increases in the option to sell it later in the secondary market. This channel is also important for those concerned with market access, as lower income households typically purchase used, not new, cars. In summary, the range of constant EV demand elasticities that we present is a deliberate simplification of demand in a complex consumer marketplace, but the intention is for readers to see the potential implications of an elasticity that falls in the range that they believe is realistic.

\subsubsection{Plug-in hybrid and battery electric vehicles}

In the projection above, we do not distinguish between battery electric vehicles and plug-in hybrid vehicles although both will likely play an important role in meeting future adoption goals. From the perspective of the buyer, the private costs of the two different powertrain technologies are different and will plausibly evolve along different trajectories. Plug-in hybrids allow for a buyer to substitute between gasoline and electricity as the primary fuel and to overcome potential range challenges that might be more important for light trucks. Furthermore, their upfront costs are closer to those of conventional vehicles as a result of a smaller battery. Yet, 
at present gasoline prices, battery electric vehicles offer lower operational costs than plug-in hybrids (under typical use) for much of the country. The relative future mix of plug-in hybrid and battery electric vehicles largely depends on how energy prices evolve (as the demand and supply of electricity shift outward) and the degree to which falling battery costs close the upfront price gap between battery electric vehicles and plug-in hybrids.

Whether the future path of adoption favors one powertrain technology over the other also has important implications for the public benefits of electric vehicles. The difference in public benefits depends primarily upon the composition of the electric grid and the relative use of electricity and gasoline by plug-in hybrids. To a lesser extent, the public benefits of battery electric vehicles also depends on whether future battery technology better overcomes efficiency losses associated low-temperature operation as discussed in Archsmith et al. (2015). Although it is difficult to predict how the grid (and particular how the marginal generating unit) will evolve over the next decade and half, Holland et al. (2020) finds that electric vehicles have become cleaner over the past decade relative to comparable conventional vehicles.

\section{Conclusion}

Policymakers view electrification of the vehicle fleet as one of the primary strategies for reducing carbon emissions and have set ambitious future adoption targets to help achieve this vision. Yet, the cost (and more broadly feasibility) of meeting those targets depends on the degree of organic growth in the future demand for EVs. Although credibly predicting future technological innovation, trends or adoption is a challenging exercise (at best), our goal in this paper is to understand underlying drivers of organic adoption that might hinder or aid progress towards the ambitious vision of a less carbon-intensive transportation sector.

In this draft, we focus upon determinants of future EV adoption: (1) the introduction of electric powertrains into the light truck segment of the EV market, and (2) the supplementary impact of future subsidies. Our scenarios suggest that both may be necessary to meet ambitious future targets, even with all but the most "aggressive" assumptions of the continuous growth of the intrinsic demand for EVs. We also observe that the intrinsic growth is substantially more important to future EV market share than government subsidies.

Intuitively, introducing an equally attractive slate of EV offerings for the roughly seventy percent of new car buyers who currently purchase SUVs, crossovers, van and trucks, has a demonstrably larger impact on the feasibility of reaching future EV targets than a subsidy. In our main scenario, we assume that the light truck market reaches "quality-parity" toward the 
end of our forecast period, at which time the suite of light truck EV offerings is viewed as equally attractive to light truck buyers as sedan EVs are to sedan buyers. This convergence drives our prediction of the market share of EV light trucks to surpass the market share of EV sedans toward the end of our study period. Yet, whether "quality-parity" is reached depends centrally on ability of automakers to design light truck EVs that capture the attention of buyers who differ from buyers who have been traditionally attracted to EV sedans and who rank different attributes as important in their purchase decisions. And, relative to the lower priced sedan market, the market for light trucks, with their attendant higher prices, might be less easy to influence with subsidies than the lower priced sedan market.

We also note that the two levers operate along different margins and have important implications for whether adoption patterns in future years are more or less balanced across regions of the country. "Quality-parity" of light truck EVs plays a role in balancing EV adoption between regions of the U.S. with relative preferences for light trucks (e.g., the Midwest) and regions with relative preferences for sedans (e.g., the West and East Coasts). In contrast, an extension of the federal subsidy tends to increase heterogeneity in adoption patterns across states as a flat subsidy has a larger impact in percentage terms on lower priced sedans than higher priced light trucks. 


\section{References}

Archsmith, James, Alissa Kendall, and David Rapson, "From Cradle to Junkyard: Assessing the Life Cycle Greenhouse Gas Benefits of Eletric Vehicles," Research in Transportation Economics, 2015, 63 (3), 397-421.

Berry, Steven, James Levinsohn, and Ariel Pakes, "Automobile Prices in Market Equilibrium," Econometrica, 1995, 63(4), 841-890.

Borenstein, Severin and Lucas W Davis, "The distributional effects of US clean energy tax credits," Tax Policy and the Economy, 2016, 30 (1), 191-234.

Chevalier, Judith and Austan Goolsbee, "Are durable goods consumers forward-looking? Evidence from college textbooks," The Quarterly Journal of Economics, 2009, 124 (4), 18531884.

Demographics Research Group, “National Population Projections," University of Virgina Weldon Cooper Center, 2018.

Goldberg, Pinelopi Koujianou, "Product Differentiation and Oligopoly in International Markets: The Case of the U.S. Automobile Industry," Econometrica, 1995, 63(4), 891-951.

_ , "The Effects of the Corporate Average Fuel Efficiency Standards in the U.S.," The Journal of Industrial Economics, March 1998, 46 (1), 1-33.

Holland, Stephen, Erin Mansur, Nicholas Muller, and Andrew Yates, "Are There Environmental Benefits from Driving Electric Vehicles? The Importance of Local Factors," American Economic Review, 2016, 106 (12), 3700-3729.

Holland, Stephen P, Erin T Mansur, Nicholas Z Muller, and Andrew J Yates, “Decompositions and policy consequences of an extraordinary decline in air pollution from electricity generation," American Economic Journal: Economic Policy, 2020, 12 (4), 244-74.

Howe, Peter D, Matto Mildenberger, Jennifer R Marlon, and Anthony Leiserowitz, "Geographic variation in opinions on climate change at state and local scales in the USA," Nature Climate Change, 2015, 5 (6), 596-603.

International Energy Agency, “Global EV Outlook 2020,” Technical Report 2020.

Li, Jing, "Compatibility and Investment in the U.S. Electric Vehicle Market," Working Paper 2017. 
Li, Shanjun, Lang Tong, Jianwei Xing, and Yiyi Zhou, “The market for electric vehicles: indirect network effects and policy design," Journal of the Association of Environmental and Resource Economists, 2017, 4 (1), 89-133.

Milovanoff, Alexandre, I Daniel Posen, and Heather L MacLean, "Electrification of lightduty vehicle fleet alone will not meet mitigation targets," Nature Climate Change, 2020, 10 (12), 1102-1107.

Muehlegger, Erich and David Rapson, "Subsidizing Mass Adoption of Electric Vehicles: Quasi-Experimental Evidence from California," Technical Report, National Bureau of Economic Research 2018.

_ and _ , "The Economics of Electric Vehicles," Technical Report, National Bureau of Economic Research 2021.

Springel, Katalin, "Network Externality and Subsidy Structure in Two-Sided Markets: Evidence from Electric Vehicle Incentives," Working Paper 2017. 


\section{Figures and Tables}

Figure 1: Moving Average EV Sales Growth

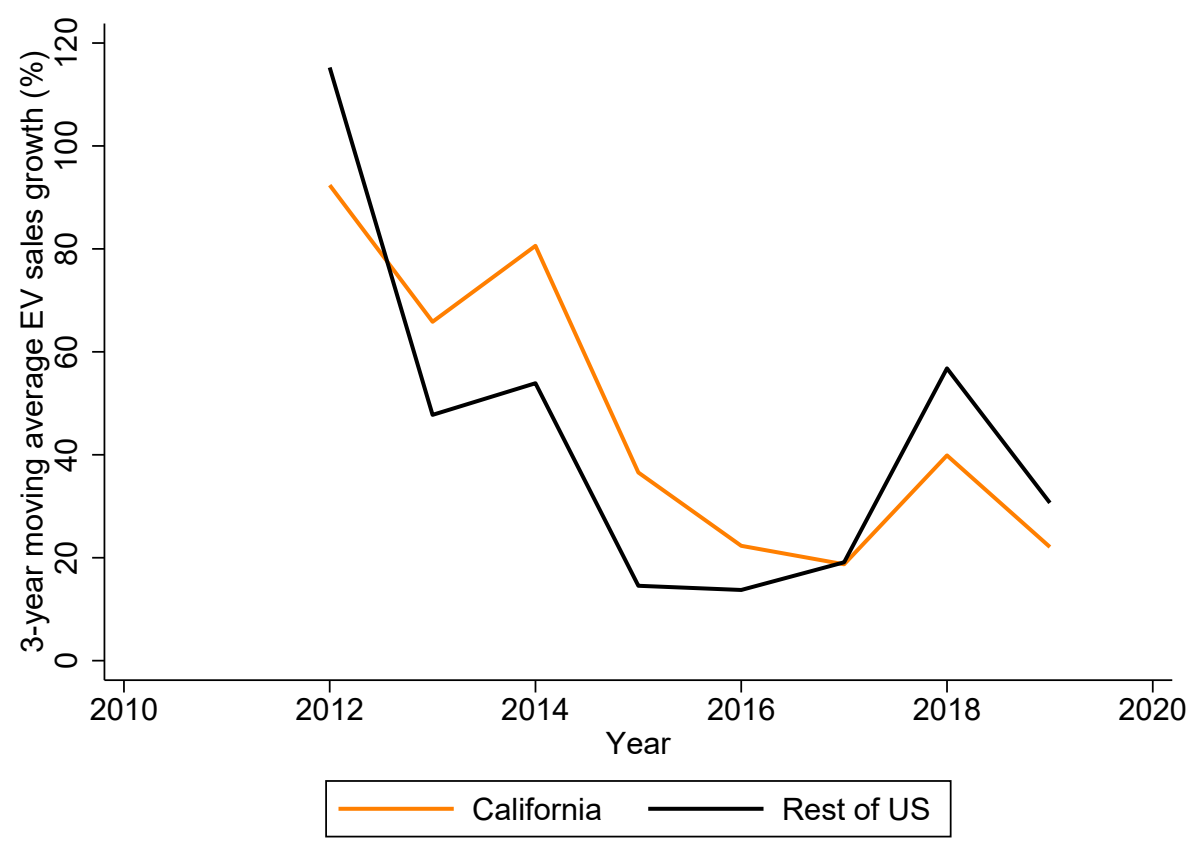

Notes: 3-year moving average growth in EV sales for California and the remainder of the United States 
Figure 2: Vehicle Type Market Shares

(a) Sedan market Share

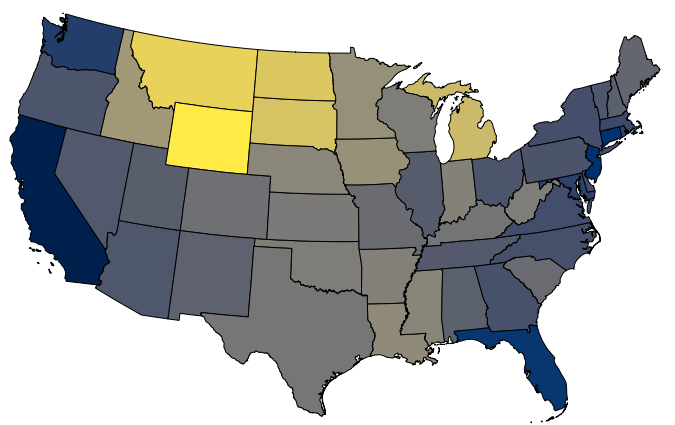

Market Share

(\% of all vehicles Sold)

$30 \% \quad 40 \% \quad 50 \%$ (b) Truck market Share

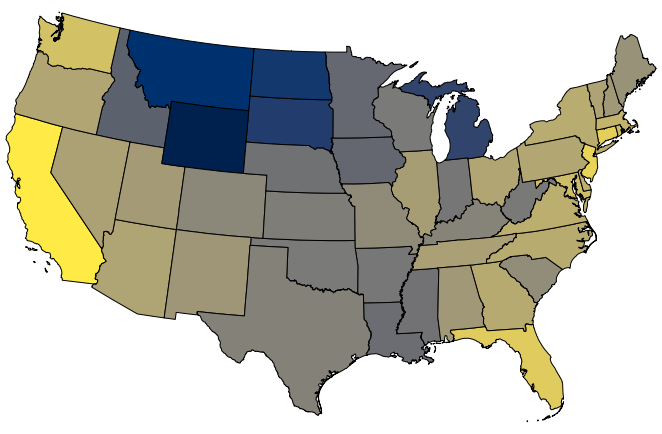

Market Share
(\% of all vehicles Sold) \% of all vehicles

(c) EV market share

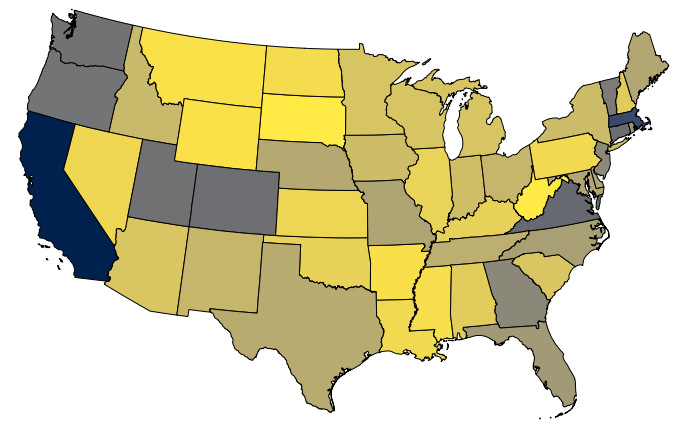

Market Share
(\% of all vehicles Sold)

$\begin{array}{llll}1 \% & 2 \% & 3 \%\end{array}$

Notes: Market share for sedans, trucks, and EVs from Maritz survey respondents in 2017 and 2018. Washington, D.C., excluded from the scale. 
Figure 3: Demographic characteristics and EV adoption
(a) Income
(b) Education
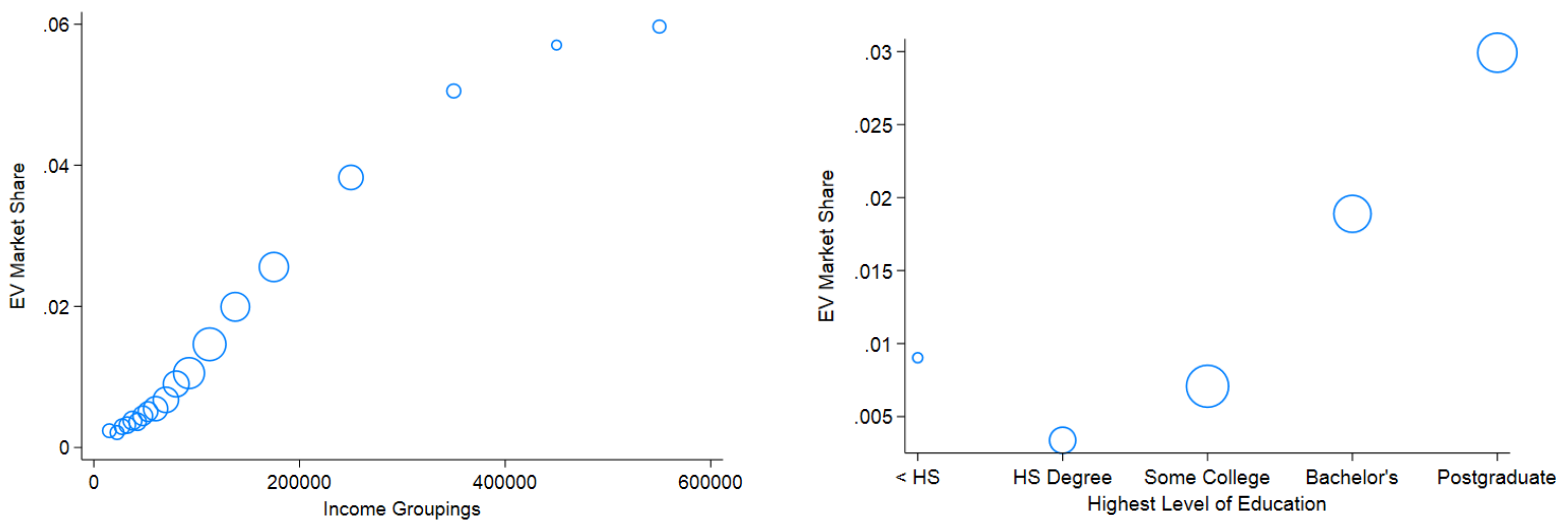

(c) Age

(d) Ethnicity
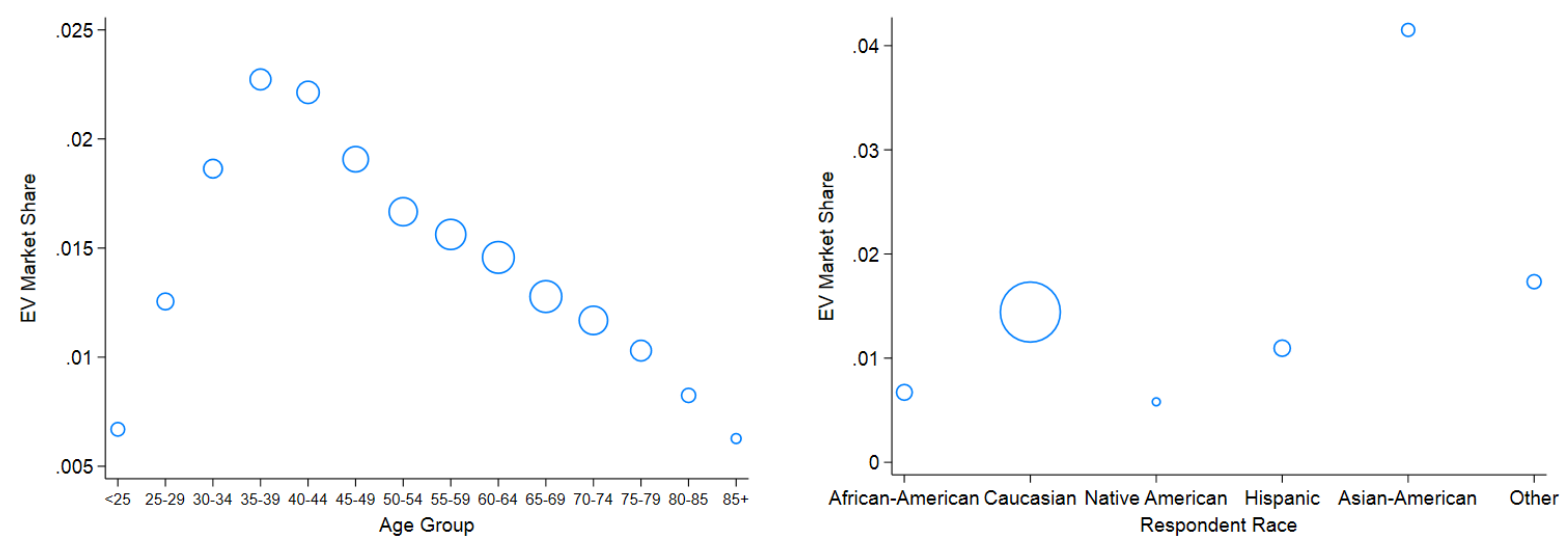

Notes: Source: Maritz. 
Figure 4: Climate Change Beliefs and Vehicle Adoption

(a) EV Adoption

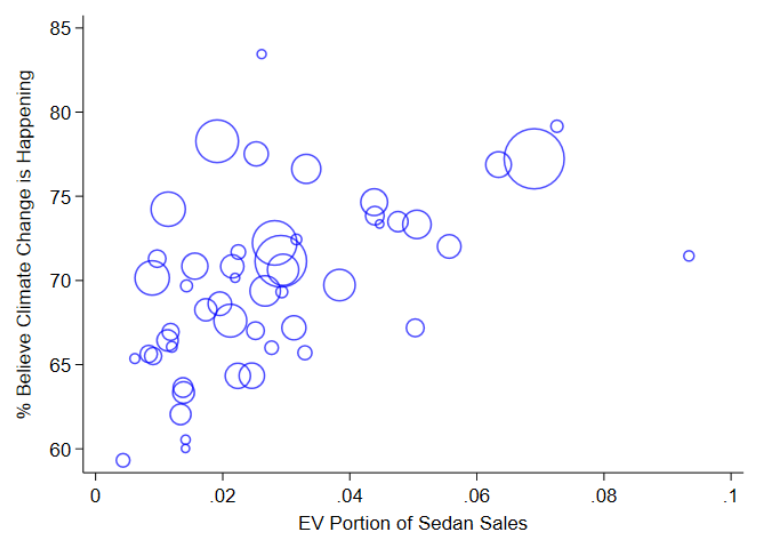

(b) Light truck Ownership

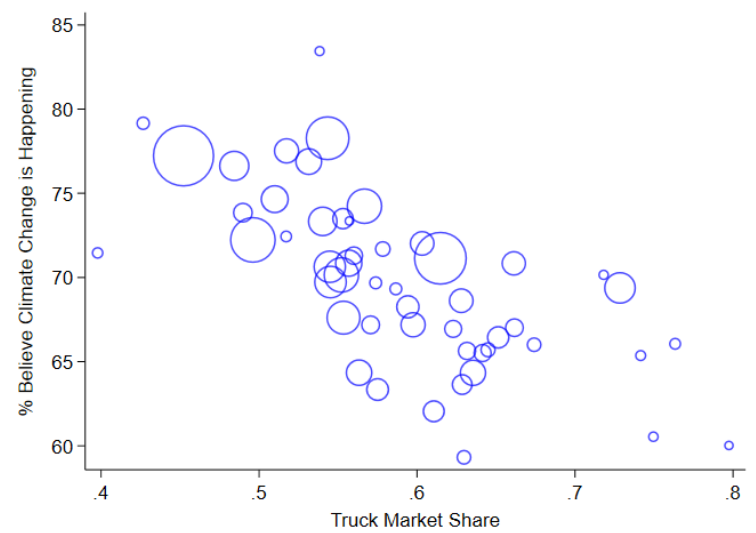

Notes: X-axes plots to the share of EVs as a fraction of total sedan sales (left-panel) and the share of light trucks as a fraction of total vehicle sales. The Y-axis plots the share of a state's population that answered affirmatively to the question "Do you believe that climate change is happening?" ( Howe et al. (2015) ) available via the Yale Climate Opinion Map, https: / / climatecommunication.yale.edu/visualizations-data/ycom-us/. The size of the circles reflect 2020 population.

Figure 5: Vehicle purchase price by vehicle class

(a) Conventional Sedan

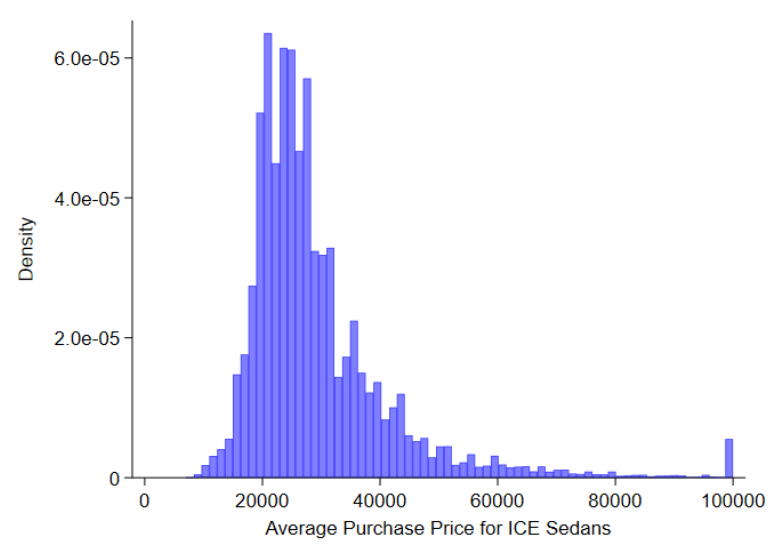

(b) SUV / Truck Ownership

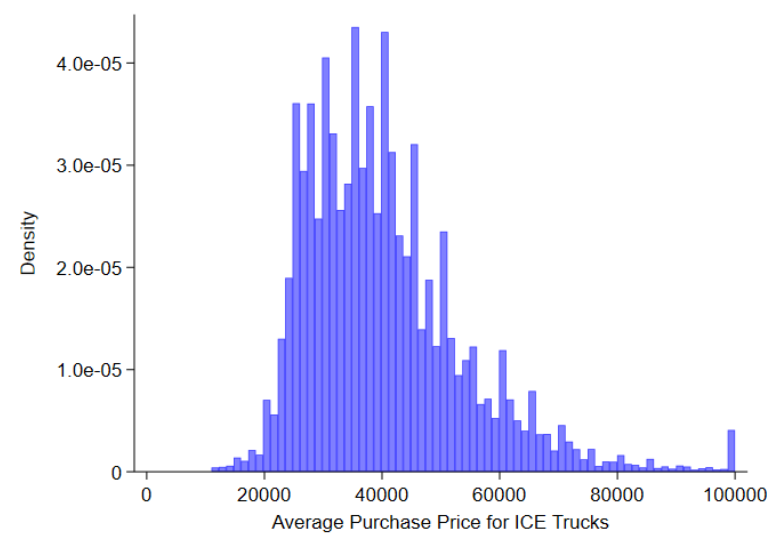

Notes: Figures plot the histograms of vehicle purchase price for conventional sedans (left-hand panel) and light trucks / SUVs (right-hand panel). Source: Maritz. 
Figure 6: Market Share Model Outline

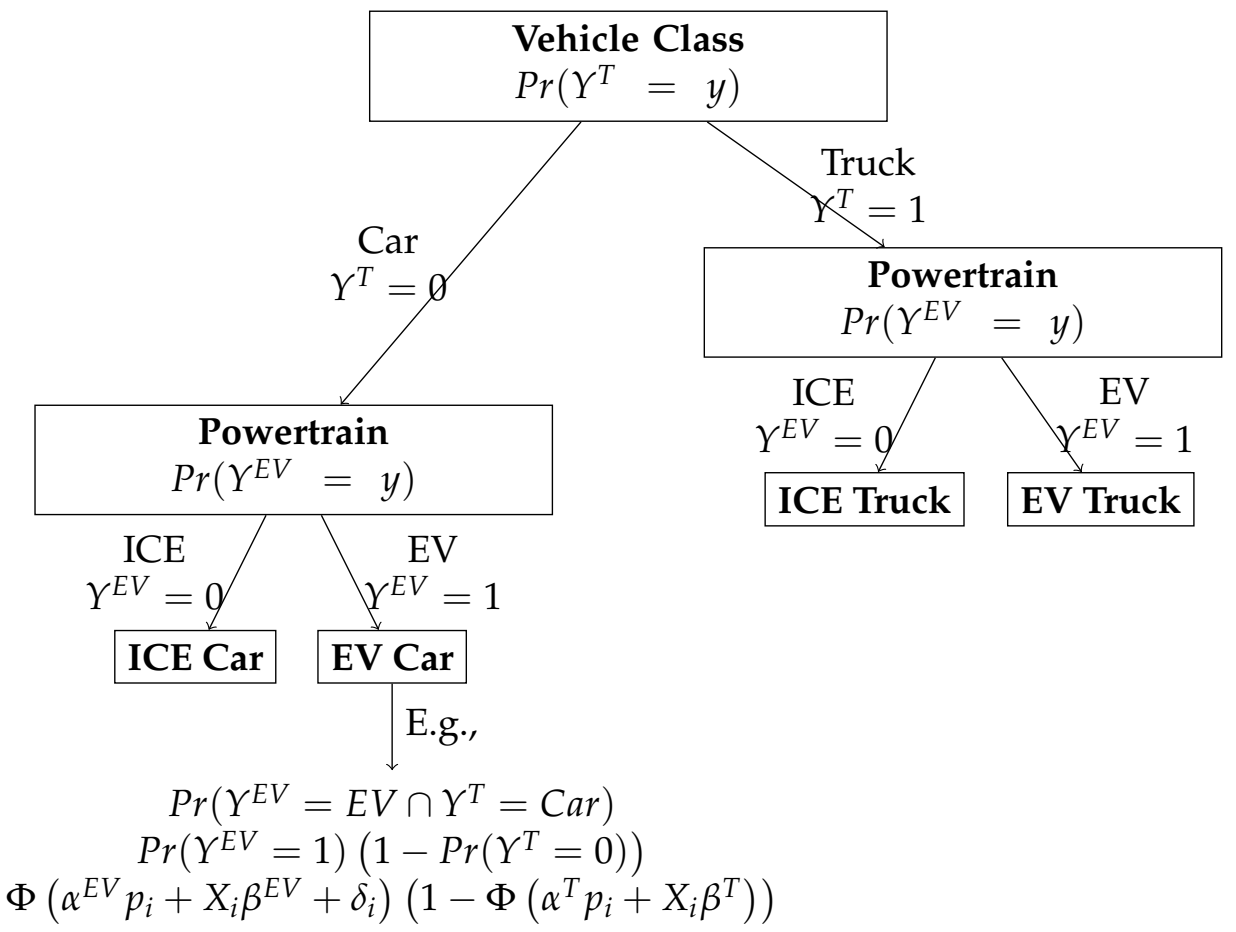

Notes: Outline of the Probit model used to predict vehicle market shares. The parameters $\alpha^{E V}$ and $\alpha^{T}$ are price effects for EVs and trucks, respectively. These parameters are fixed prior to estimation of the model to achieve an assumed price elasticity of market share at the average consumer. The parameter $\delta_{i}$ in the Powertrain component is the intrinsic preference for electric vehicles and is constant across all consumers in a given year. Is it zero in 2020 and increases over time so BAU EV market share (absent price effects and demographic change) matches the assumed growth rate. 


\section{Figure 7: Future BAU EV Sales Growth Scenarios}

(a) Forecast Scenarios through 2035

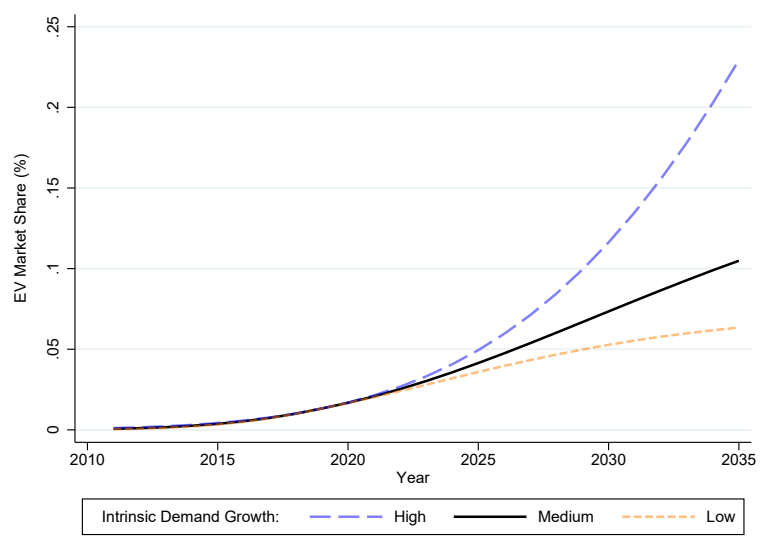

(b) Backcasting actual growth rates to present

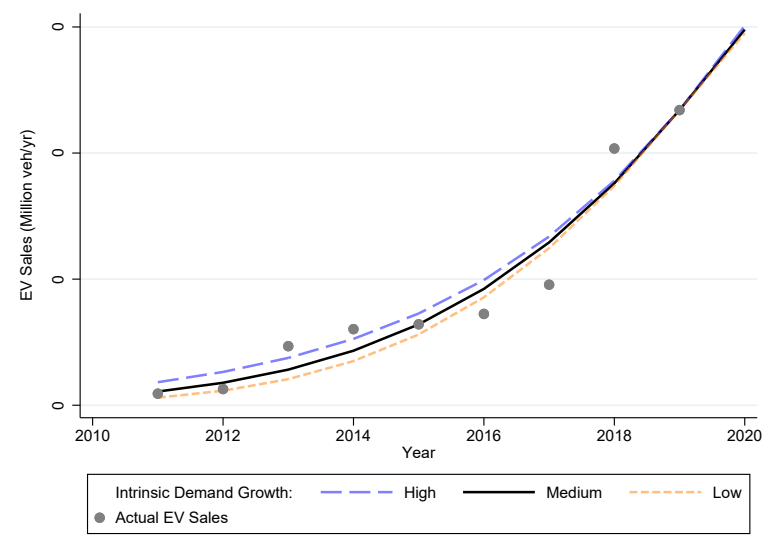

(c) Forecast Scenarios YOY EV Sales Growth

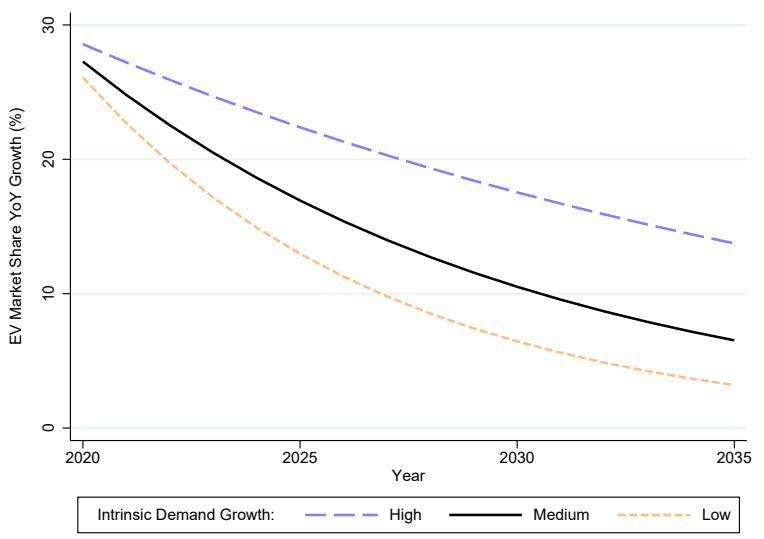

Notes: Forecast scenarios for business as usual (BAU) EV sales growth. Absent other intervention, EV sales grow at 30\% year-over year in 2020. That rate of increase decreases by 5\%,10\%, and 15\% in the high, baseline, and low EV growth scenarios. The full path of EV market share through 2035 is shown in Panel (a). EV sales following this pattern are backcasted to 2010 and constrasted with actual sales (blue dots) in Panel (b). The rate of year-over-year sales growth in each scenario is shown in Panel (c). 
Figure 8: Purchase behavior model predictions

(a) Vehicle Class Model

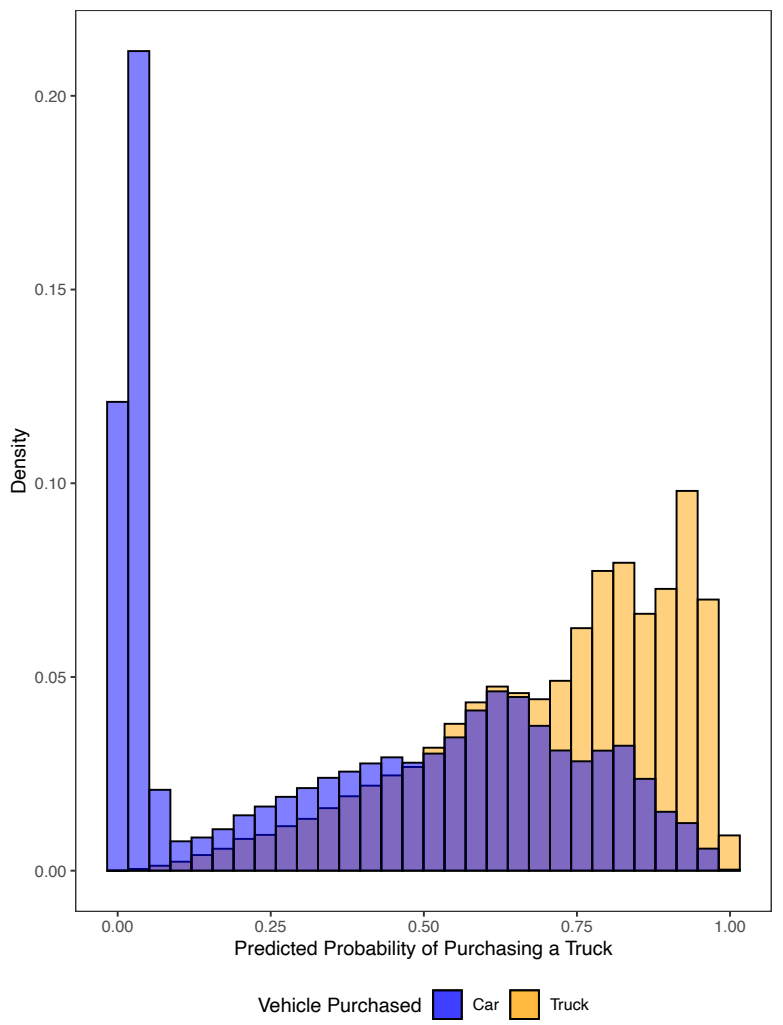

(b) Powertrain Model

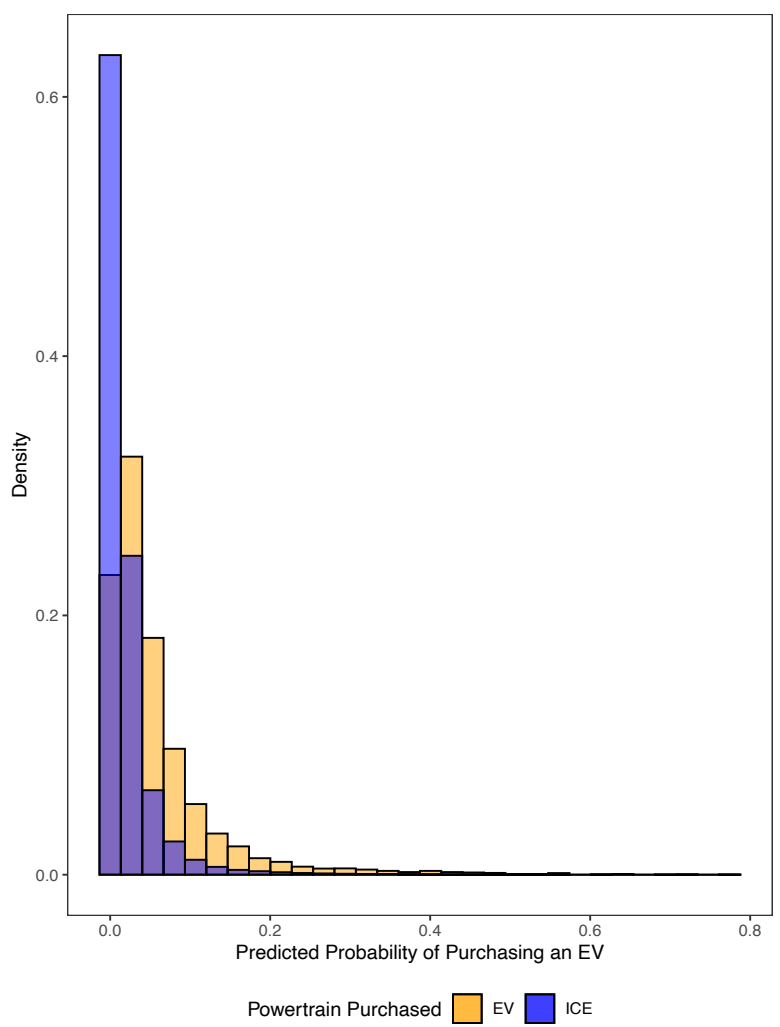

Histogram of the predicted purchase probability by the actual purchase behavior of consumers in the Maritz survey. Densities are weighted by survey weights. Perfect predictions would result in a single blue bar at zero and a single orange bar at 1 . 
Figure 9: Sales by vehicle type, with and without subsidies

(a) No Subsidy - High EV Demand Growth

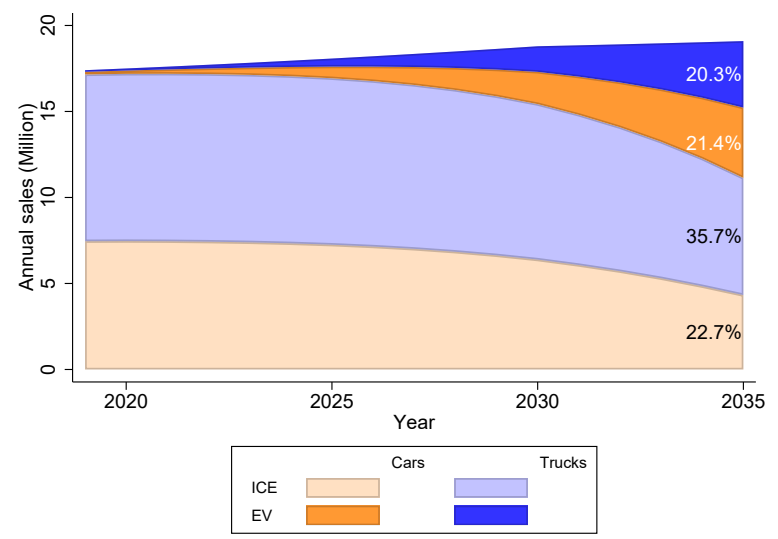

(c) No Subsidy - Medium EV Demand Growth

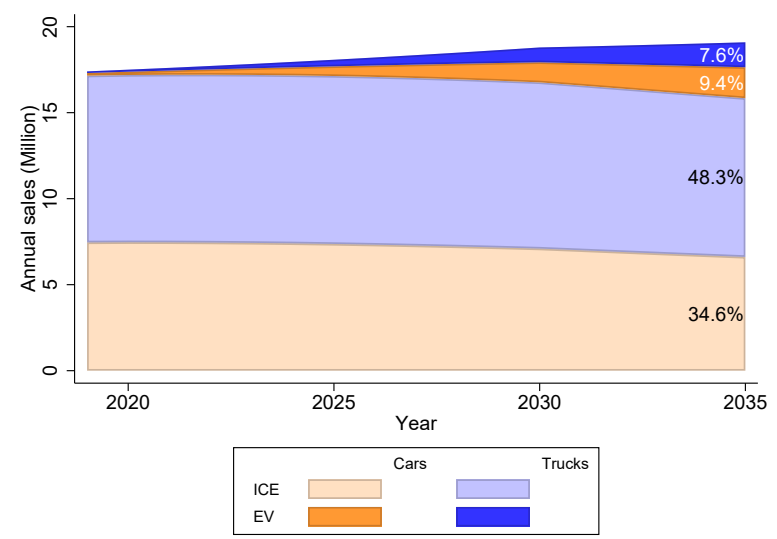

(e) No Subsidy - Low EV Demand Growth

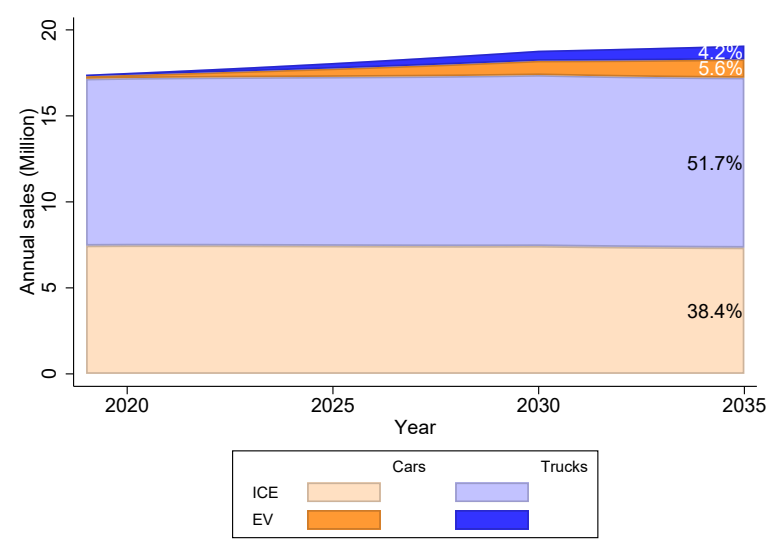

(b) Federal Subsidy - High EV Demand Growth

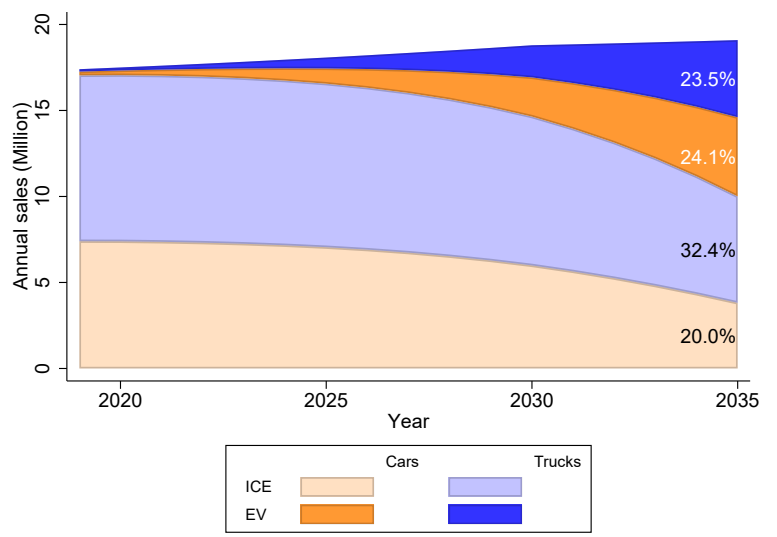

(d) Federal Subsidy - Medium EV Demand Growth

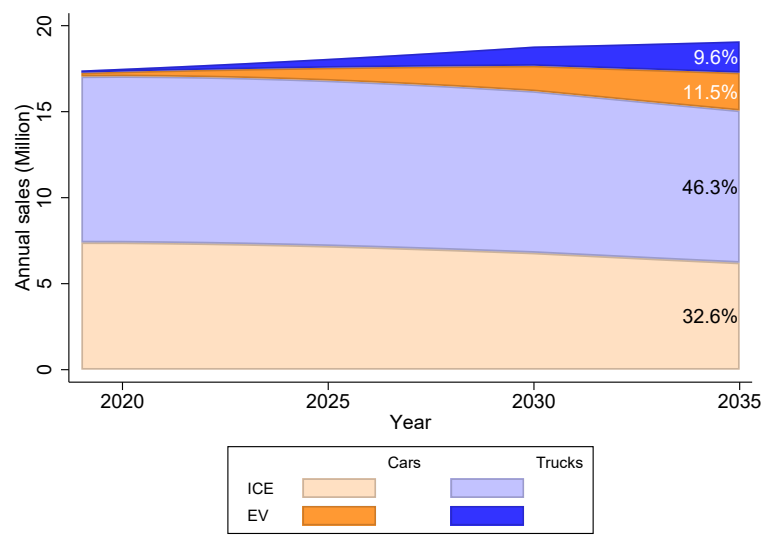

(f) Federal Subsidy - Low EV Demand Growth

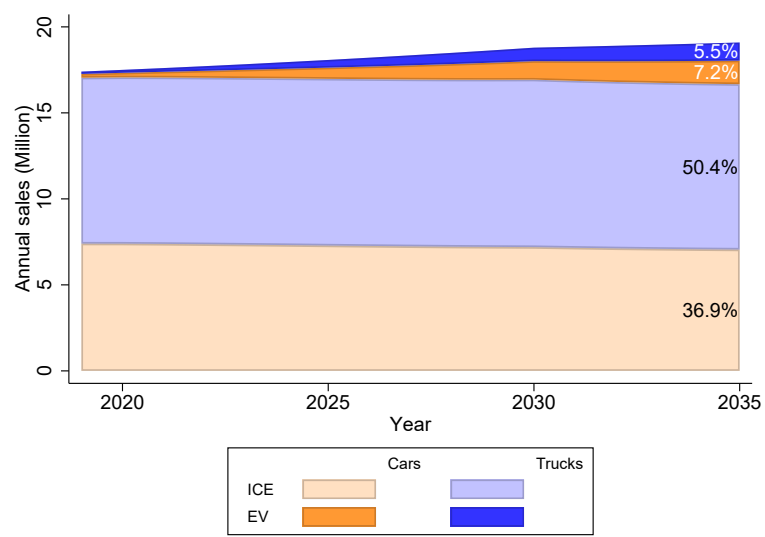

Notes: Intrinsic EV demand growth is 30\% in 2020 and the growth rate declines by 5\% (High Growth), 10\% (Med Growth), or 15\% (Low Growth) each year. 
Figure 10: Subsidy Bill and EV Market Share in 2035

(a) EV Cars and Trucks Available

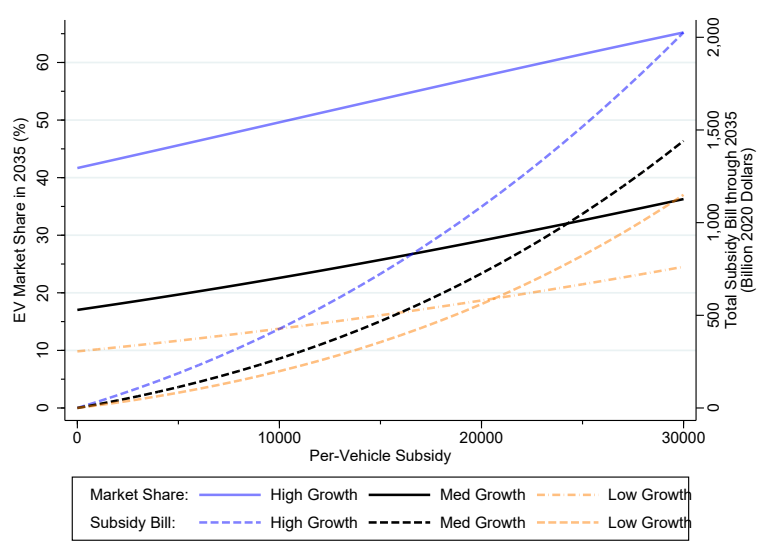

(b) EV Cars Only

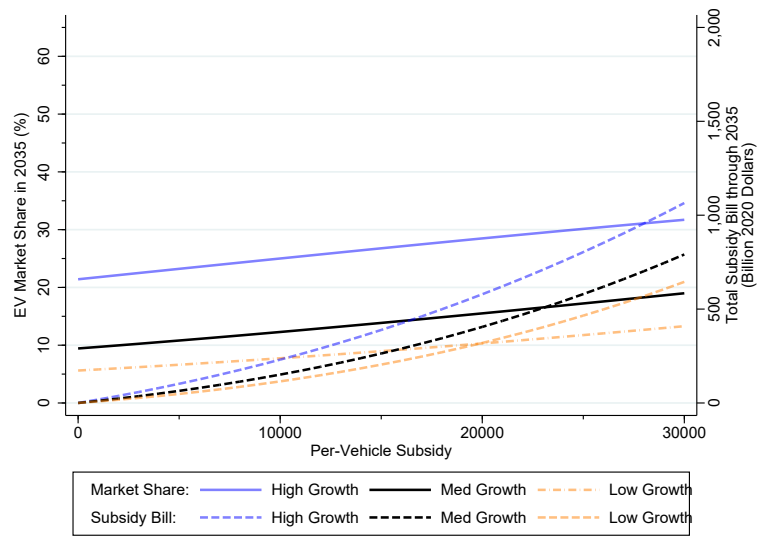

Notes: Total subsidy bill and 2035 EV market share for a range of per-vehicle subsidies. Present value of the total subsidy bill through 2035 in 2020 dollars, at a 3\% discount rate. Scenarios assume intrinsic EV demand growth is 30\% in 2020 and declines by $5 \%$ (High Growth), 10\% (Med Growth), or 15\% (Low Growth) each year.

Figure 11: Subsidy Bill to Achieve EV Market Share Targets in 2035

(a) EV Cars and Trucks Available

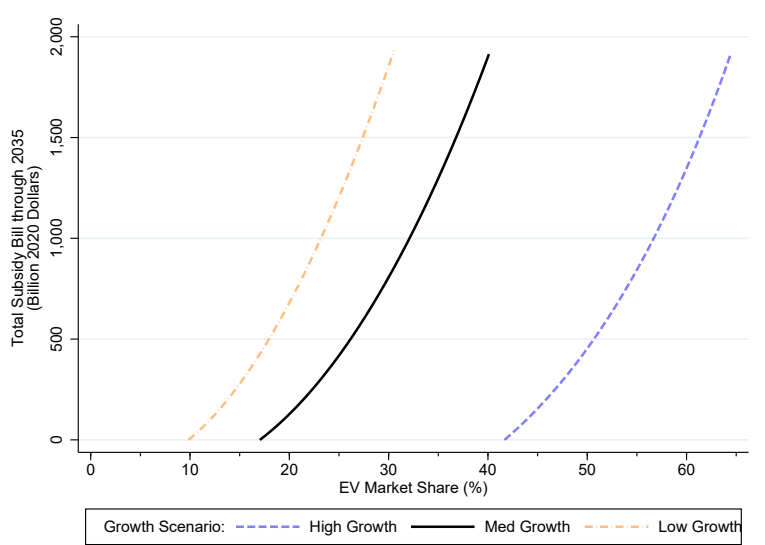

(b) EV Cars Only

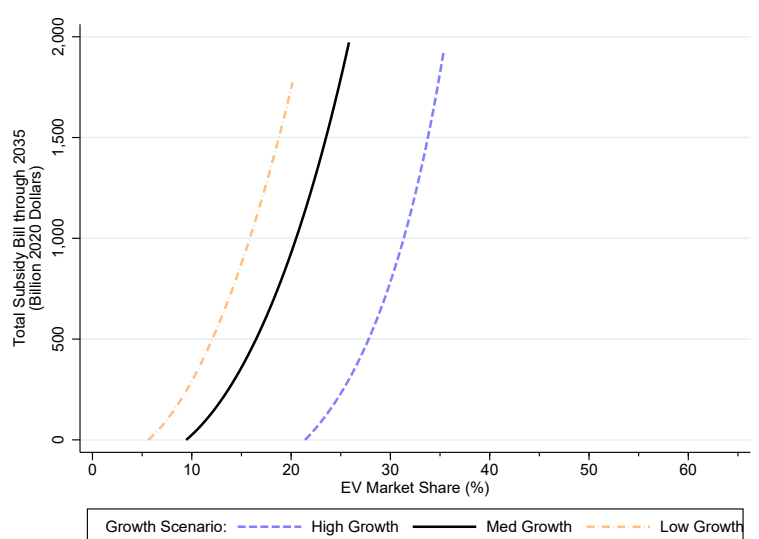

Notes: Relationship between EV market share in 2035 and the total subsidy bill required to achieve that market share. Present value of the total subsidy bill through 2035 in 2020 dollars, at a 3\% discount rate. Scenarios assume intrinsic EV demand growth is 30\% in 2020 and declines by 5\% (High Growth), 10\% (Med Growth), or 15\% (Low Growth) each year. 
Figure 12: EV adoption by state, with and without light truck EVs, 2035

(a) All EVs

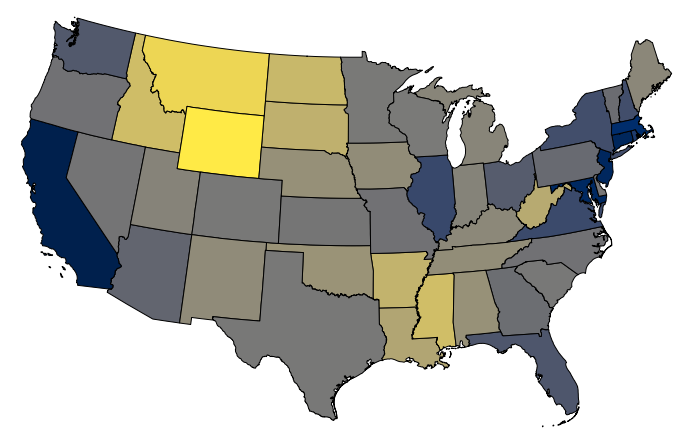

EV Sales (\% of all vehicles)

$9 \% 12 \% 15 \% 18 \%$

(b) Sedan EVs

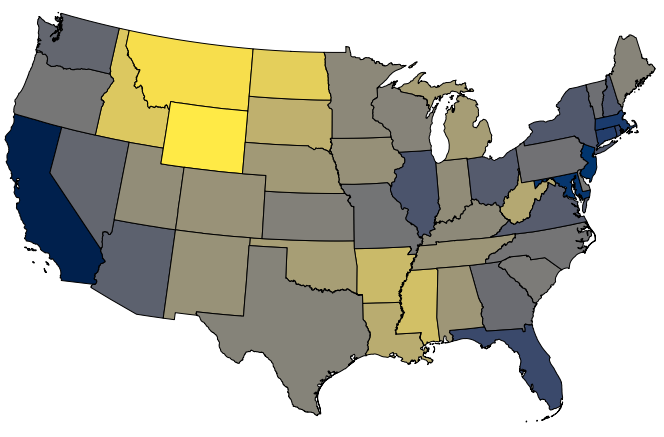

EV Sales (\% of all vehicles)

$5 \% 7.5 \% 10 \% 12.5 \%$ (c) Light Truck EVs

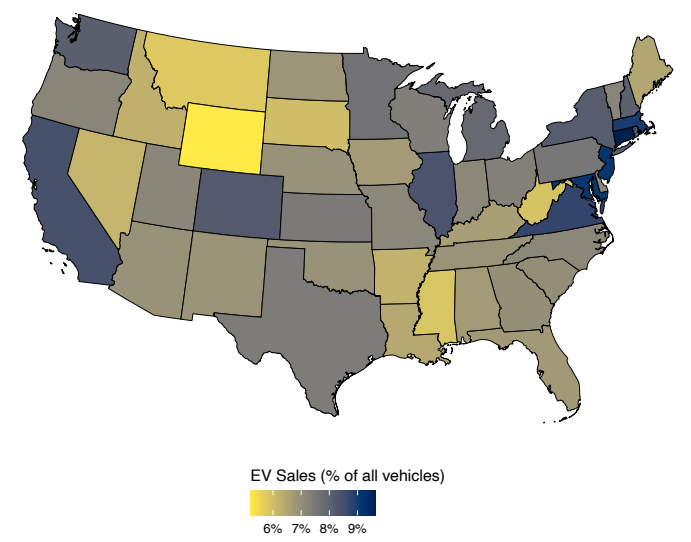

Notes: Forecast EV market share by state in 2035. Assumes medium EV demand growth, EV sedans reach price parity with comparable ICEs in 2030, EV Trucks reach price parity in 2040, and no Federal EV subsidies. Washington, D.C., excluded from the scale. 
Figure 13: Heterogeneity in EV penetration across states, 2035

(a) No Subsidy - High EV Demand Growth

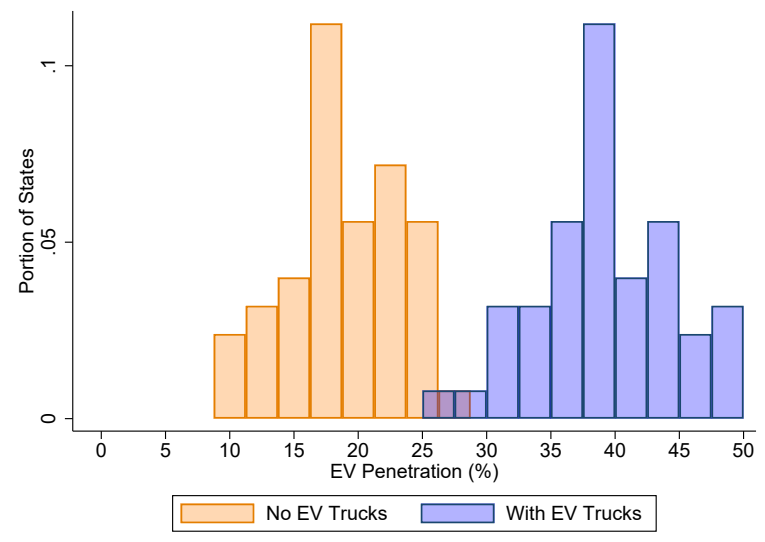

(b) No Subsidy - Medium EV Demand Growth

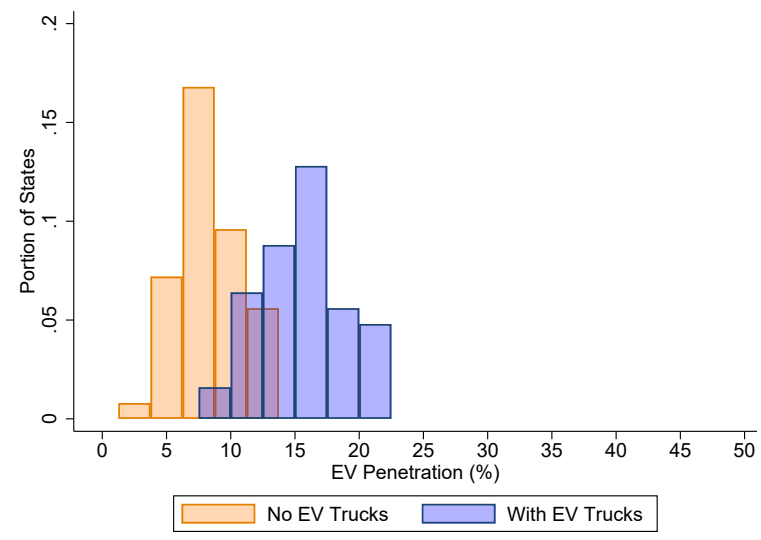

(c) No Subsidy - Low EV Demand Growth

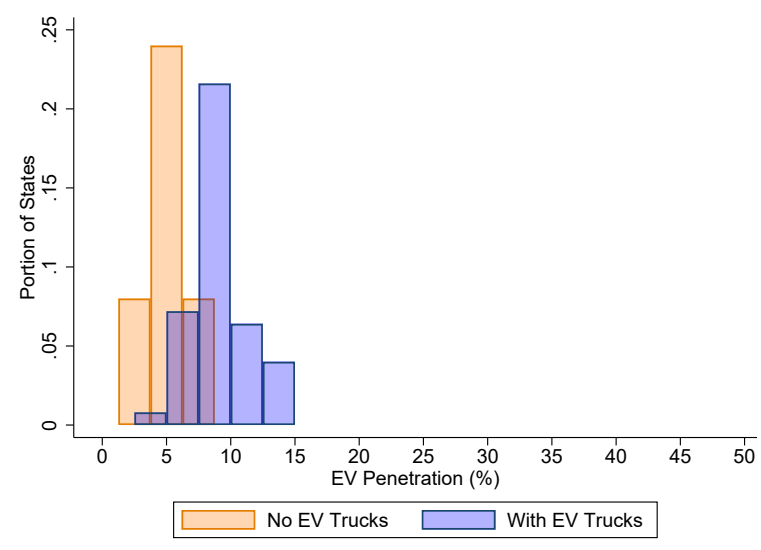

Notes: Distribution of the state-by-state EV market share accounting for EV sedans only (orange) and EV sedans and trucks (blue). Excludes Washington, D.C. Intrinsic EV demand growth is 30\% in 2020 and the growth rate declines by 5\% (High Growth), 10\% (Med Growth), or 15\% (Low Growth) each year. 
Figure 14: Additional Impact of Subsidies on EV Penetration in 2035

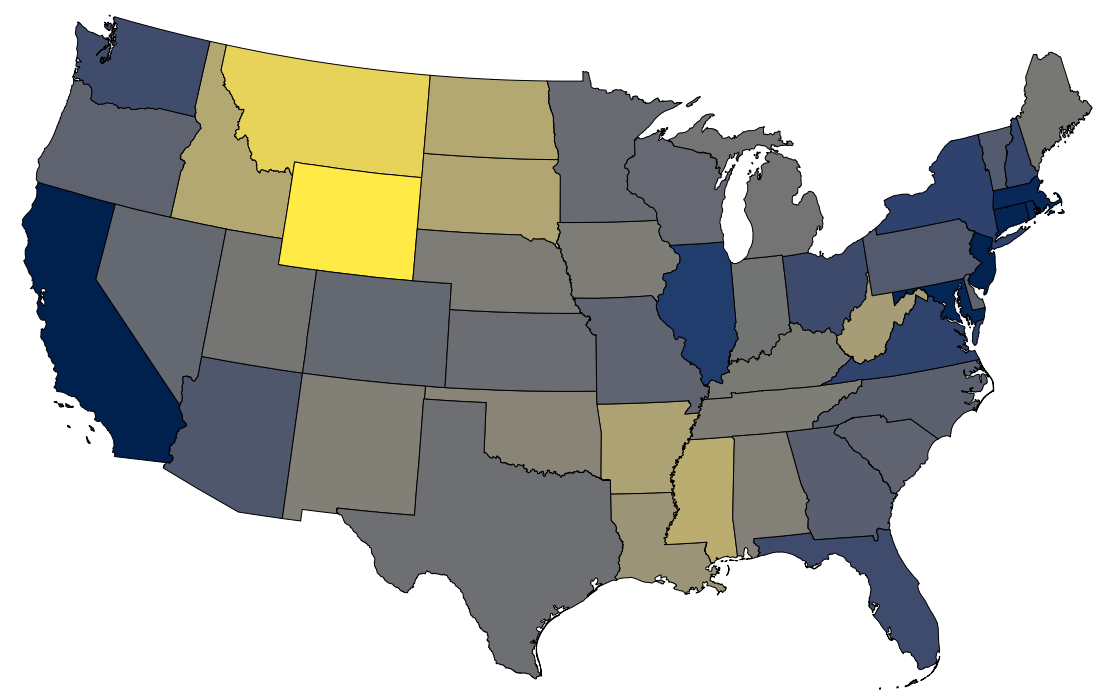

EV Sales (\% of all vehicles)

$3 \% 3.5 \% 4 \% 4.5 \%$

Notes: Additional market share of EVs in each state in 2035 induced by a $\$ 7,500$ per-vehicle subsidy. Assumes intrinsic demand growth of $30 \%$ in 2020 that declines at a rate of $10 \%$ per year, EV cars reach cost parity with their ICE counterparts in 2030 and trucks in 2040. Washington, D.C. excluded from the scale. 
Figure 15: Sensitivity of Market Shares to Price Elasticity

(a) Low Price Response

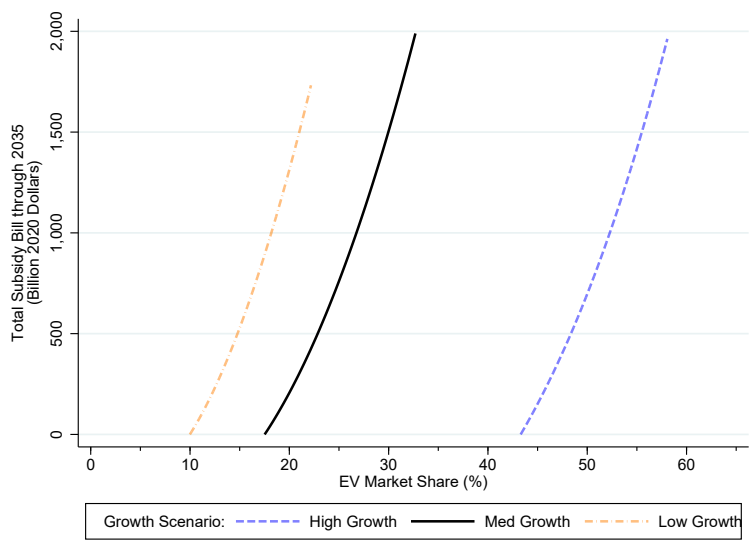

(c) High Price Response

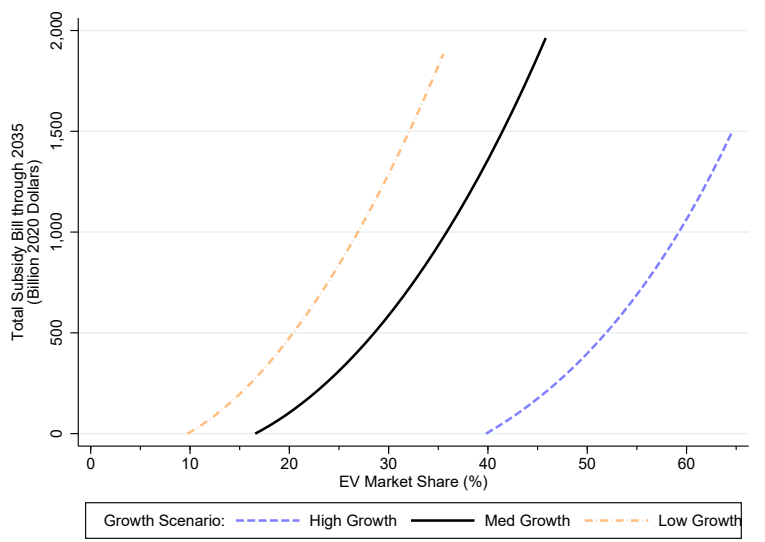

(b) Medium Price Response

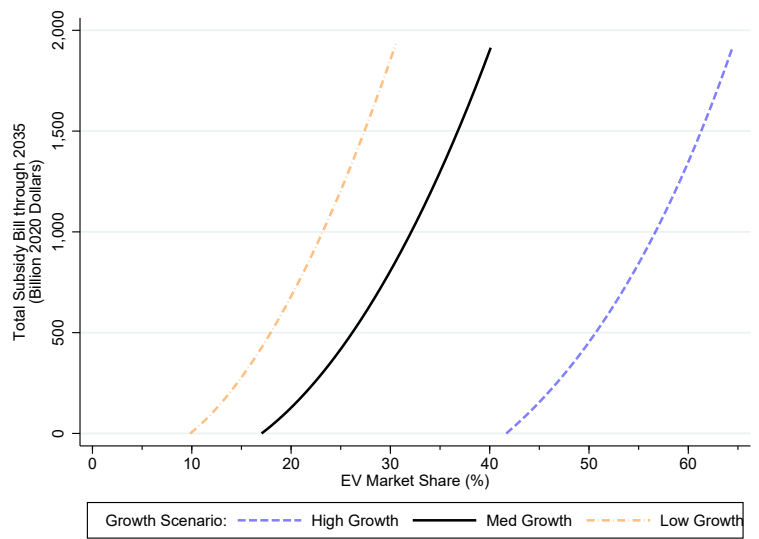

(d) Income-Dependent Price Response

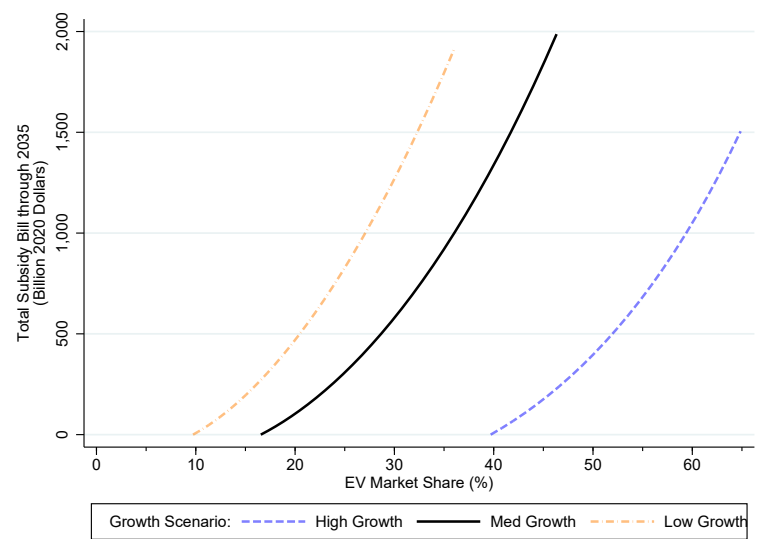

Notes: Relationship between EV market share in 2035 and the total subsidy bill required to achieve that market share across a range of potential consumer price responsiveness. Price responsiveness ( $\alpha^{E V}$ in the EV market share model) is selected so that the price elasticity of EV market share for the average vehicle purchaser is $-1,-2$, or -3 (Low, Medium, and High price-responsiveness, respectively). Medium price responsiveness is assumed in other figures. Panel (d) assumes consumers in the top income quartile have low price responsiveness, with the average consumer in this group having an elasticity of -1 , and all other consumers have high price responsiveness, with the average consumer in this group having an elasticity of -3 . The vertical axis shows the present value of the total subsidy bill in 2035 in 2020 dollars, at a 3\% discount rate and the horizontal axis shows the resulting EV market share in 2035. Scenarios assume intrinsic EV demand growth is 30\% in 2020 and declines by 5\% (High Growth), 10\% (Med Growth), or 15\% (Low Growth) each year. 
Table 1: State Level PEV Incentives

\begin{tabular}{|c|c|c|c|}
\hline & & & State Level Electric Vehicle Policies \\
\hline State & Policy & Max. Subsidy & Notes \\
\hline Arkansas & Alternative Fuels Vehicle and Rebate Program & 2,500 & $\begin{array}{l}\text { Rebate for the cost difference between an EV and } \\
\text { similar ICE. Ran out of funds in } 4 / 2018\end{array}$ \\
\hline California & CVRP & 2,500 & Means tested rebate program for EV purchases \\
\hline Colorado & Innovative Motor Vehicle Credit & 5,000 & Tax credit of $\$ 5,000$ for $\mathrm{EVs}$ \\
\hline Connecticut & CHEAPR & 3,000 & $\begin{array}{l}\text { Rebate which varies by the electric range of the } \\
\text { vehicle }\end{array}$ \\
\hline D.c. & Excise Tax Credit for Plug-in Vehicles & $6 \%$ & $\begin{array}{l}\text { Vehicle excise tax waiver which can reach up to } \\
6 \% \text { of the purchase price }\end{array}$ \\
\hline Delaware & DE Clean Vehicle Rebate Program & 3,500 & Varies by price and electric engine type \\
\hline Louisiana & Alternative Fuel Vehicle Tax Credit & 3,000 & Tax credit available for BEV purchases only \\
\hline Maryland & Excise Tax Credit for Plug-in Electric Vehicles & 3,000 & $\begin{array}{l}\text { Tax credit starting in July } 2017 \text { which varies by } \\
\text { battery size }\end{array}$ \\
\hline Massachusetts & MOR-EV & 2,500 & $\begin{array}{l}\text { Rebate which varies by the purchase price of the } \\
\text { vehicle and electric engine type }\end{array}$ \\
\hline New Jersey & Sales Tax Exemption & $6.87 \%$ & $\begin{array}{l}\text { Sales tax exemption which applies to only BEV } \\
\text { purchases }\end{array}$ \\
\hline New York & Drive Clean Rebate & 2,000 & $\begin{array}{l}\text { Varies by purchase price and electric range of } \\
\text { vehicle }\end{array}$ \\
\hline Oregon & Clean Vehicle Rebate Program & 2,500 & $\begin{array}{c}\text { Begins in } 2018 \text { for BEVs only. Varies by battery size } \\
\text { and purchase price }\end{array}$ \\
\hline Pennsylvania & Alternative Fuel Vehicle Rebate & 2,000 & Rebate varies by battery size and purchase price. \\
\hline Rhode Island & DRIVE Rebate Program & 2,500 & Rebate varies by battery size and ends in July 2017 \\
\hline Texas & Alternative Fuel Vehicle Rebate & 2,500 & $\begin{array}{l}\text { Rebate starts in Sept. 2018. Does not apply to } \\
\text { Tesla vehicles }\end{array}$ \\
\hline shington & Alternative Fuels Tax Credit & $6.50 \%$ & Ends in July 2017 and varies by battery size \\
\hline
\end{tabular}


Table 2: Predicted Market Share (2020-2035)

(a) No Subsidies - High Growth

\begin{tabular}{rrrr}
\hline Year & EV Sedans & EV Trucks & EV Total \\
\hline 2025 & $3.7 \%$ & $2.6 \%$ & $6.3 \%$ \\
2030 & $10.0 \%$ & $7.9 \%$ & $17.9 \%$ \\
2035 & $21.4 \%$ & $20.3 \%$ & $41.7 \%$ \\
\hline
\end{tabular}

(c) No Subsidies - Medium Growth

\begin{tabular}{rrrr}
\hline Year & EV Sedans & EV Trucks & EV Total \\
\hline 2025 & $3.1 \%$ & $2.1 \%$ & $5.2 \%$ \\
2030 & $6.2 \%$ & $4.6 \%$ & $10.8 \%$ \\
2035 & $9.4 \%$ & $7.6 \%$ & $17.0 \%$ \\
\hline
\end{tabular}

(e) No Subsidies - Low Growth

\begin{tabular}{rrrr}
\hline Year & EV Sedans & EV Trucks & EV Total \\
\hline 2025 & $2.7 \%$ & $1.8 \%$ & $4.5 \%$ \\
2030 & $4.4 \%$ & $3.1 \%$ & $7.5 \%$ \\
2035 & $5.6 \%$ & $4.2 \%$ & $9.8 \%$ \\
\hline
\end{tabular}

(b) Continued Federal Subsidies - High Growth

\begin{tabular}{rrrr}
\hline Year & EV Sedans & EV Trucks & EV Total \\
\hline 2025 & $4.9 \%$ & $3.5 \%$ & $8.4 \%$ \\
2030 & $12.1 \%$ & $9.9 \%$ & $22.1 \%$ \\
2035 & $24.1 \%$ & $23.5 \%$ & $47.6 \%$ \\
\hline
\end{tabular}

(d) Continued Federal Subsidies - Medium Growth

\begin{tabular}{rrrr}
\hline Year & EV Sedans & EV Trucks & EV Total \\
\hline 2025 & $4.1 \%$ & $2.9 \%$ & $7.0 \%$ \\
2030 & $7.9 \%$ & $6.0 \%$ & $13.8 \%$ \\
2035 & $11.5 \%$ & $9.6 \%$ & $21.1 \%$ \\
\hline
\end{tabular}

(f) Continued Federal Subsidies - Low Growth

\begin{tabular}{rrrr}
\hline Year & EV Sedans & EV Trucks & EV Total \\
\hline 2025 & $3.6 \%$ & $2.5 \%$ & $6.1 \%$ \\
2030 & $5.7 \%$ & $4.2 \%$ & $9.9 \%$ \\
2035 & $7.2 \%$ & $5.5 \%$ & $12.7 \%$ \\
\hline
\end{tabular}

Notes: Intrinsic demand growth of 30\% in 2020 declines at a rate of 5\%, 10\% and $15 \%$ per year in the high, medium and low growth cases, respectively. EV cars reach cost parity with their ICE counterparts in 2030 and trucks in 2040. 
Table 3: EV Market Share in 2035 by State

\begin{tabular}{lrrr}
\hline \multirow{2}{*}{ State } & \multicolumn{3}{c}{ Intrinsic Demand Growth } \\
\cline { 2 - 4 } District Of Columbia & $64.8 \%$ & $39.9 \%$ & $29.3 \%$ \\
California & $54.8 \%$ & $26.5 \%$ & $16.6 \%$ \\
Connecticut & $54.1 \%$ & $26.0 \%$ & $16.2 \%$ \\
New Jersey & $54.3 \%$ & $25.9 \%$ & $16.1 \%$ \\
Maryland & $53.8 \%$ & $25.8 \%$ & $16.1 \%$ \\
Massachusetts & $53.5 \%$ & $25.4 \%$ & $15.8 \%$ \\
Hawaii & $53.2 \%$ & $25.4 \%$ & $15.9 \%$ \\
Rhode Island & $52.6 \%$ & $24.3 \%$ & $14.8 \%$ \\
Illinois & $50.7 \%$ & $23.2 \%$ & $14.2 \%$ \\
Virginia & $50.1 \%$ & $23.0 \%$ & $14.1 \%$ \\
& & & \\
.. & & & \\
Louisiana & $40.0 \%$ & $15.8 \%$ & $8.9 \%$ \\
West Virginia & $39.2 \%$ & $15.4 \%$ & $8.7 \%$ \\
South Dakota & $38.3 \%$ & $15.0 \%$ & $8.5 \%$ \\
Arkansas & $38.5 \%$ & $14.8 \%$ & $8.3 \%$ \\
North Dakota & $38.0 \%$ & $14.5 \%$ & $8.1 \%$ \\
Alaska & $36.9 \%$ & $14.2 \%$ & $8.0 \%$ \\
Idaho & $37.9 \%$ & $14.2 \%$ & $7.8 \%$ \\
Mississippi & $37.4 \%$ & $14.1 \%$ & $7.8 \%$ \\
Montana & $33.8 \%$ & $12.4 \%$ & $6.8 \%$ \\
Wyoming & $31.7 \%$ & $11.1 \%$ & $6.0 \%$ \\
\hline
\end{tabular}

Notes: Intrinsic demand growth of 30\% in 2020 that declines at a rate of $10 \%$ per year, EV cars reach cost parity with their ICE counterparts in 2030 and trucks in 2040. Market shares assume a $\$ 7,500$ per-vehicle subsidy on EVs. 
Table 4: EV Market Share in 2035 by Model Assumptions

\begin{tabular}{|c|c|c|c|c|}
\hline Average & Price & \multicolumn{3}{|c|}{ Intrinsic EV Demand Growth } \\
\hline EV Price & Response & High & Medium & Low \\
\hline$\$ 40,000$ & High & $36.5 \%$ & $14.6 \%$ & $8.4 \%$ \\
\hline$\$ 40,000$ & Medium & $39.2 \%$ & $15.5 \%$ & $8.8 \%$ \\
\hline$\$ 40,000$ & Low & $42.0 \%$ & $16.7 \%$ & $9.4 \%$ \\
\hline$\$ 37,500$ & High & $39.3 \%$ & $16.2 \%$ & $9.5 \%$ \\
\hline$\$ 37,500$ & Medium & $41.3 \%$ & $16.8 \%$ & $9.6 \%$ \\
\hline$\$ 37,500$ & Low & $43.1 \%$ & $17.4 \%$ & $9.9 \%$ \\
\hline$\$ 35,000^{*}$ & High & $42.0 \%$ & $18.1 \%$ & $10.8 \%$ \\
\hline$\$ 35,000 *$ & Medium & $43.1 \%$ & $18.0 \%$ & $10.5 \%$ \\
\hline$\$ 35,000^{*}$ & Low & $44.0 \%$ & $18.0 \%$ & $10.3 \%$ \\
\hline$\$ 32,500$ & High & $44.9 \%$ & $20.0 \%$ & $12.1 \%$ \\
\hline$\$ 32,500$ & Medium & $45.3 \%$ & $19.4 \%$ & $11.5 \%$ \\
\hline$\$ 32,500$ & Low & $45.2 \%$ & $18.7 \%$ & $10.8 \%$ \\
\hline$\$ 30,000$ & High & $47.6 \%$ & $22.1 \%$ & $13.8 \%$ \\
\hline$\$ 30,000$ & Medium & $47.1 \%$ & $20.8 \%$ & $12.5 \%$ \\
\hline$\$ 30,000$ & Low & $46.1 \%$ & $19.4 \%$ & $11.3 \%$ \\
\hline$\$ 27,500$ & High & $50.7 \%$ & $24.3 \%$ & $15.3 \%$ \\
\hline$\$ 27,500$ & Medium & $49.3 \%$ & $22.3 \%$ & $13.5 \%$ \\
\hline$\$ 27,500$ & Low & $47.2 \%$ & $20.2 \%$ & $11.8 \%$ \\
\hline
\end{tabular}

Notes: Comparison of EV market share in 2035 across model assumptions and average EV prices. Average price is the average price paid for all EVs in 2035. * denotes the average price where EVs are at approximate parity with comparable ICEs. Price responses are measured at the consumer with the average valuation and are selected so the price elasticity of market share for that consumer is -3 (High), -2 (Medium), or -1 (Low). Intrinsic demand growth of $30 \%$ in 2020 declines at a rate of $5 \%, 10 \%$ and $15 \%$ per year in the high, medium and low growth cases, respectively.

Table 5: Total Subsidy Bill to Achieve Target EV Market Shares in 2035

\begin{tabular}{clll}
\hline 2035 Target EV & \multicolumn{3}{c}{ Intrinsic EV Demand Growth } \\
Market Share & High & Medium & Low \\
\hline $20 \%$ & $\$ 0.0 \mathrm{~B}$ & $\$ 140.2 \mathrm{~B}$ & $\$ 710.4 \mathrm{~B}$ \\
$35 \%$ & $\$ 0.0 \mathrm{~B}$ & $\$ 1,356.1 \mathrm{~B}$ & $\$ 2,735.5 \mathrm{~B}$ \\
$50 \%$ & $\$ 481.3 \mathrm{~B}$ & $\$ 3,562.5 \mathrm{~B}$ & $\mathrm{~N} / \mathrm{A}$
\end{tabular}

Notes: Comparison of the total subsidy bill required to achieve EV market share targets in 2035. Subsidy bills in billions of 2020 dollars with a 3\% discount rate. The subsidy is assumed a constant between 2020 and 2035. Intrinsic demand growth of 30\% in 2020 declines at a rate of 5\%,10\% and $15 \%$ per year in the high, medium and low growth cases, respectively. No subsidy less than the price of an EV results in the target market share of $50 \%$ under the low EV demand growth scenario. 